\title{
Microneedle patch for the ultrasensitive quantification of protein biomarkers in interstitial fluid
}

\author{
Zheyu Wang ${ }^{1,6}$, Jingyi Luan', ${ }^{1,6}$ Anushree Seth ${ }^{1}$, Lin Liu', Minli You', Prashant Gupta', Priya Rathi', \\ Yixuan Wang', Sisi Cao', Qisheng Jiang', Xiao Zhang ${ }^{2,3}$, Rohit Gupta ${ }^{1}$, Qingjun Zhou',

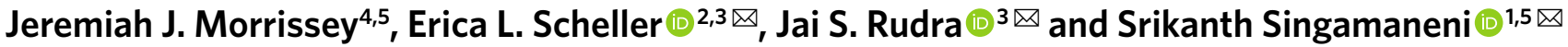

\begin{abstract}
The detection and quantification of protein biomarkers in interstitial fluid is hampered by challenges in its sampling and analysis. Here we report the use of a microneedle patch for fast in vivo sampling and on-needle quantification of target protein biomarkers in interstitial fluid. We used plasmonic fluor-an ultrabright fluorescent label-to improve the limit of detection of various interstitial fluid protein biomarkers by nearly 800 -fold compared with conventional fluorophores, and a magnetic backing layer to implement conventional immunoassay procedures on the patch and thus improve measurement consistency. We used the microneedle patch in mice for minimally invasive evaluation of the efficiency of a cocaine vaccine, for longitudinal monitoring of the levels of inflammatory biomarkers, and for efficient sampling of the calvarial periosteum-a challenging site for biomarker detection-and the quantification of its levels of the matricellular protein periostin, which cannot be accurately inferred from blood or other systemic biofluids. Microneedle patches for the minimally invasive collection and analysis of biomarkers in interstitial fluid might facilitate point-of-care diagnostics and longitudinal monitoring.
\end{abstract}

nterstitial fluid (ISF) - compared with other peripheral biofluids such as saliva, sweat and tears-is a particularly rich source of soluble bioanalytes including proteins, peptides, metabolites and nucleic acids that exhibits close correlation with blood ${ }^{1-6}$. It also represents the locoregional biomolecular composition of specific tissues of interest, such as within the tumour microenvironment ${ }^{7}$. Simple and effective methods that enable comprehensive analysis of ISF can lead to transformative advances in biodiagnostic technologies that are not only minimally invasive and pain free, but also ideally suited for point-of-care and resource-limited settings ${ }^{8-10}$. Extraction of ISF followed by ex vivo analysis ${ }^{11}$ has not been widely embraced in preclinical or clinical applications, largely because of (1) difficulty in extracting ISF, which is time consuming and requires bulky instruments ${ }^{12-14}$ and (2) the small amount of ISF that can be extracted using current technology, making comprehensive analysis challenging ${ }^{15}$. For example, microneedle-assisted extraction of ISF yields about $2 \mu \mathrm{l}$ of biofluid from $4 \mathrm{~cm}^{2}$ of human skin even after 20 min of vacuum suction, which is insufficient for comprehensive proteomic and metabolomic analysis ${ }^{15}$. Indeed, in preclinical settings (for example, when using small animal models), to measure the concentration of target biomarkers, it is common to pool ISF from multiple subjects to obtain sufficient material, which inevitably masks the subject-to-subject biological variability ${ }^{16}$.

In contrast to ISF extraction, microneedles functionalized with biorecognition elements can specifically capture target biomarkers in ISF, which can be followed by ex vivo analysis ${ }^{17,18}$. Direct exposure of microneedles to ISF allows the biorecognition elements on the microneedle to capture target biomarkers in situ, thus offer- ing a promising technology for simple and efficient biodetection. However, physiological concentrations of the protein biomarkers in the ISF are usually lower compared to those in blood ${ }^{4,19}$. Moreover, analyte-antibody binding kinetics are deteriorated due to the dense tissue environment, which results in slower diffusion of target biomolecules to the sensor surface (that is, the microneedle surface), further lowering the probability of analyte capture and consequent signal intensity corresponding to the analyte. These challenges exacerbate the difficulty of detection of protein biomarkers in interstitial fluid. Despite the recent advances in multiplexed detection of biomarkers ${ }^{20}$, the sensitivities of existing microneedle-based analytical methods are insufficient to detect (or quantify) most ISF protein biomarkers, which limits the development potential for diagnostic tests based on ISF biomarker levels. Most previous reports are limited to mice that have been intravenously injected with high concentrations of recombinant target markers as pseudo models, or to biomolecules present at relatively high levels (micrograms per millilitre in blood $)^{17}$. Finally, existing microneedle-based in vivo sampling and detection methods are limited to qualitative analysis in which the target biomarker concentration is represented as relative fluorescence intensity, absorbance value or normalized relative quantity ${ }^{18,20,21}$. This limitation precludes quantitative comparisons of the biomarker concentrations across different experiments and across different laboratories for biomedical research and decreases opportunities for standardization of the cut-off values for clinical biomarkers.

Here we demonstrate ultrasensitive and quantitative measurement of target protein biomarkers in ISF through microneedle-based

'Department of Mechanical Engineering and Materials Science, Institute of Materials Science and Engineering, Washington University in St Louis, St Louis, MO, USA. ${ }^{2}$ Department of Medicine, Division of Bone and Mineral Diseases, Washington University in St Louis, St Louis, MO, USA. ${ }^{3}$ Department of Biomedical Engineering, Washington University in St Louis, St Louis, MO, USA. ${ }^{4}$ Department of Anesthesiology, Washington University in St Louis, St Louis, MO, USA. ${ }^{5}$ Siteman Cancer Center, Washington University School of Medicine, St Louis, MO, USA. ${ }^{6}$ These authors contributed equally: Zheyu Wang, Jingyi Luan.凶e-mail: scheller@wustl.edu; srudra22@wustl.edu; singamaneni@wustl.edu 
in vivo sampling and subsequent on-needle analysis. To improve the sensitivity of our microneedle-based immunoassay, we used an ultrabright fluorescent nanolabel, termed plasmonic fluor, which improved the limit of-detection of various ISF protein biomarkers by nearly 800 -fold compared with conventional fluorophores and markedly decreased the sampling time. Moreover, by harnessing the bilayer design of the microneedle, we were able to replicate conventional immunoassay procedures on microneedle patches, including a calibration curve based on 'standard micropatches'. Using a series of mouse models, we demonstrate that the microneedle patch can be used for ultrasensitive and quantitative monitoring of various protein biomarkers using a simple stick-and-peel process. First, we probed the efficiency of a cocaine vaccine by monitoring cocaine-specific antibodies in dermal ISF. Second, we demonstrated sensitive detection and longitudinal monitoring of inflammatory biomarker levels in mice after induction of endotoxin-mediated shock. Last, we validated the application of the microneedle patch in the detection and quantification of the matricellular protein periostin (POSTN) in the calvarial periosteum-a challenging site for detection-using both control wild-type (WT) and periostin knockout $\left(\right.$ Post $\left.^{K O}\right)$ mice. The minimally invasive microneedle patch obviates the need for destruction of target tissues and repeated drawing of blood over a short period, which can cause poor patient compliance or potential death of experimental mice in preclinical settings. This ultrasensitive biodetection technology may enable the broad use of microneedle patches for biomarker capture and analysis.

\section{Results}

Design and fabrication of the microneedle patch. The biodetection technology introduced in this study relies on microneedles functionalized with biorecognition elements (such as antibodies) that penetrate the stratum corneum (or periosteum) and selectively capture protein biomarkers in the local ISF in a concentration-dependent manner. Subsequently, the microneedle patch is peeled off from the skin and the protein biomarkers bound on the microneedles are quantified by an ultrasensitive fluorescent immunoassay implemented ex vivo (Fig. 1a). For efficient capture of target biomarkers in vivo, microneedles are required to exhibit high protein-antibody binding ability, high mechanical strength and biocompatibility. Owing to its low cost, easy processability and hydrophobic nature, polystyrene is widely used for microtitre plates in biomedical research and clinical diagnostics. Affinity reagents such as capture antibodies and blocking proteins can be efficiently immobilized on the polystyrene surface owing to the hydrophobic interactions between polystyrene and the nonpolar residues on the proteins. Thus, we used polystyrene for the fabrication of the microneedles, which were subsequently coated with capture antibodies to enable specific binding of the target biomarkers.

The microneedle patch was fabricated using a silicone mould via two successive drop-casting steps (Fig. 1b). Polystyrene solution $(25 \% \mathrm{w} / \mathrm{v}$ in dichloromethane) was first cast on a silicone mould and the solvent was allowed to evaporate slowly under ambient conditions. Subsequently, a backing layer comprised of the mixture of polystyrene and magnetic $\left(\mathrm{Fe}_{3} \mathrm{O}_{4}\right)$ nanoparticles was formed on top of the pristine polystyrene layer. Incorporation of the magnetic nanoparticles in the backing layer is important to ensure that the microneedle patches stay at the bottom of a microtitre plate that is placed over a magnet during subsequent immunoassay procedures (Fig. 1b,c and Supplementary Fig. 1). This bilayer design overcomes the low throughput and poor reproducibility of patch-by-patch handling (that is, incubation and wash steps performed manually one patch at a time), making the microneedle-based assay highly consistent, fast and reproducible (Fig. 1d and Supplementary Fig. 2). The patch is comprised of an array of microneedles with a centre-to-centre distance of $600 \mu \mathrm{m}$ (Fig. 1e). Each microneedle is conical in shape with a $4 \mu \mathrm{m}$ radius of curvature at the tip, a diameter of $300 \mu \mathrm{m}$ at the base and around $600 \mu \mathrm{m}$ in height (Fig. 1e and Supplementary Fig. 3). To assess the density and uniformity of antibody coating on polystyrene microneedles, we coated the microneedles with biotinylated anti-mouse IgG followed by blocking with bovine serum albumin (BSA). Subsequently, the microneedle patches were exposed to dye (LT680)-labelled streptavidin, resulting in a strong and uniform fluorescent signal along the entire length of the microneedle, indicating the uniform coating of the antibodies on the polystyrene microneedle surface (Fig. 1f). By contrast, microneedles coated with BSA which was subsequently exposed to LT680-streptavidin exhibited extremely weak fluorescence intensity (155-fold lower, signal close to that of a pristine microneedle) (Supplementary Fig. 4), suggesting low auto-fluorescence of the BSA and polystyrene microneedle surface.

Plasmonic fluor-linked immunosorbent assay on the microneedle patch. Conventional sandwich enzyme-linked immunosorbent assay (ELISA) involves an enzymatic reaction that results in the formation of a soluble coloured product in an analyte concentration-dependent manner. While highly standardized and routinely implemented in microtitre plates comprised of identical sampling wells, this approach is unsuitable for the microneedle patches due to (1) the relatively low sensitivity stemming from the limited sampling surface area (analyte is present only on the micro-sized needles in real sampling situation), making the quantification of low-abundant analytes challenging; and (2) the soluble nature of the coloured product, which masks spatial variations in the amount of analyte bound across the patch, eliminating the possible spatial multiplexing capability. Therefore, existing approaches are limited to pseudo mouse models involving high amounts of target analytes, which do not represent their true pathological and physiological concentrations. To overcome these challenges, we used a fluorophore-linked immunosorbent assay (FLISA) that relies on plasmonic fluor as an ultrabright and highly specific fluorescent nanolabel. Plasmonic fluor comprises a gold nanorod (AuNR) coated with fluorophores (800CW) and a universal biological recognition element (for example, biotin $)^{22}$. BSA is used as a scaffold to assemble all of these functional elements, as well as to resist non-specific binding (Fig. 2a and Supplementary Fig. 5; a detailed description of plasmonic fluor is provided in the Supplementary Information). Siloxane copolymer is used as a spacer layer between the AuNR and the fluorophores to avoid metal-induced fluorescence quenching ${ }^{23}$. Transmission electron microscopy (TEM) images of the plasmonic fluor confirmed the presence of a thin organic layer around the AuNRs (polymer and BSA conjugate) with an overall thickness of about $6.3 \pm 1.1 \mathrm{~nm}$ (all values are expressed as mean \pm s.d.) (Fig. $2 \mathrm{~b}$ and Supplementary Fig. 6). Binding of plasmonic fluor-800CW to strepdavidin-800CW coated onto the bottom of a microtitre well resulted in a nearly 1,400-fold enhancement of the ensemble fluorescence intensity (Fig. 2c and Supplementary Fig. 7).

To test the applicability of plasmonic fluor as an ultrabright biolabel for use on a microneedle patch, we used mouse interleukin 6 (IL-6), a pro-inflammatory cytokine, as a representative protein biomarker. Conventional FLISA involves a standard sandwich immunoassay format, where the capture antibody is immobilized on the surface of the microneedle, recognition and capture of analyte (IL-6), binding of biotinylated detection antibody to the captured analyte, and exposure to streptavidin-fluorophore (800CW in this study), which binds to the biotin on the detection antibody with very high affinity. In contrast to conventional FLISA, plasmonic fluor-linked immunosorbent assay ( $p$-FLISA) involves the use of plasmonic fluor instead of a conventional fluorophore as the fluorescent label. To determine the improvement in sensitivity and limit of-detection (LOD, defined as mean $+3 \sigma$ of the blank) of p-FLISA compared with FLISA, serial dilutions of IL-6 of known 
a

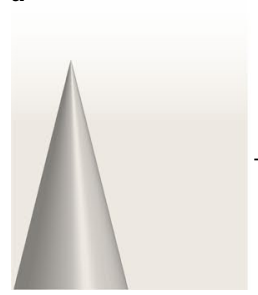

Polystyrene microneedle

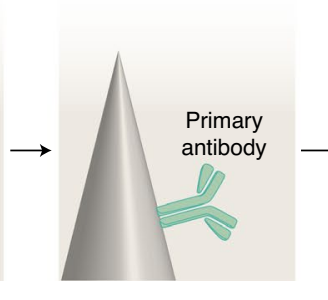

Capture antibody

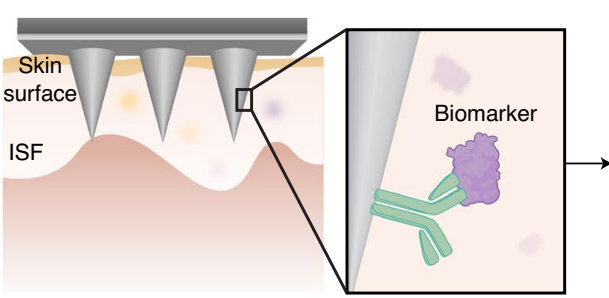

Selectively capture protein biomarkers in ISF

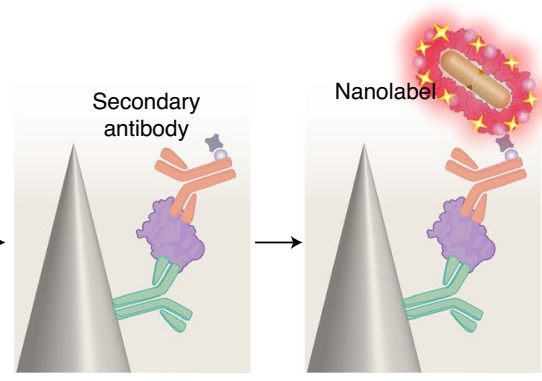

Detection antibody
Ultrabright nanolabel (plasmonic fluor)

In situ sampling

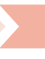

b

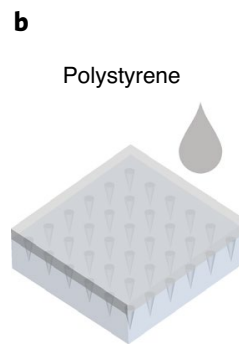

d

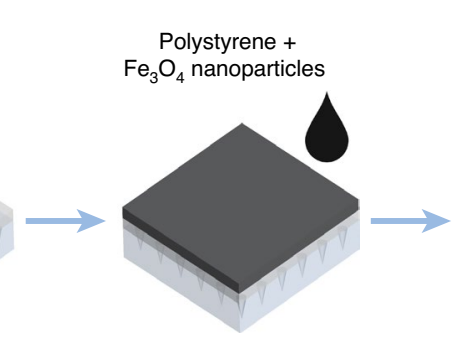

Incubation or washing
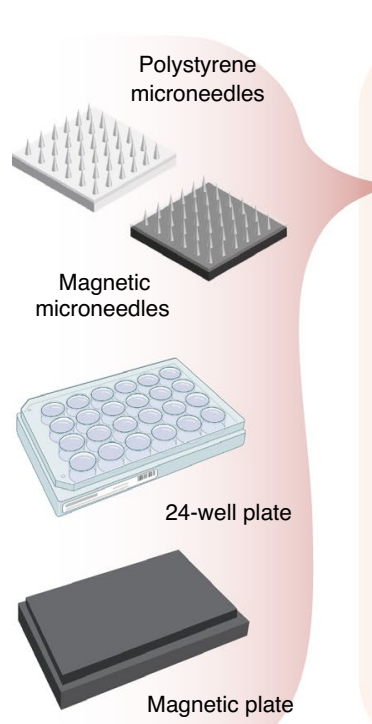
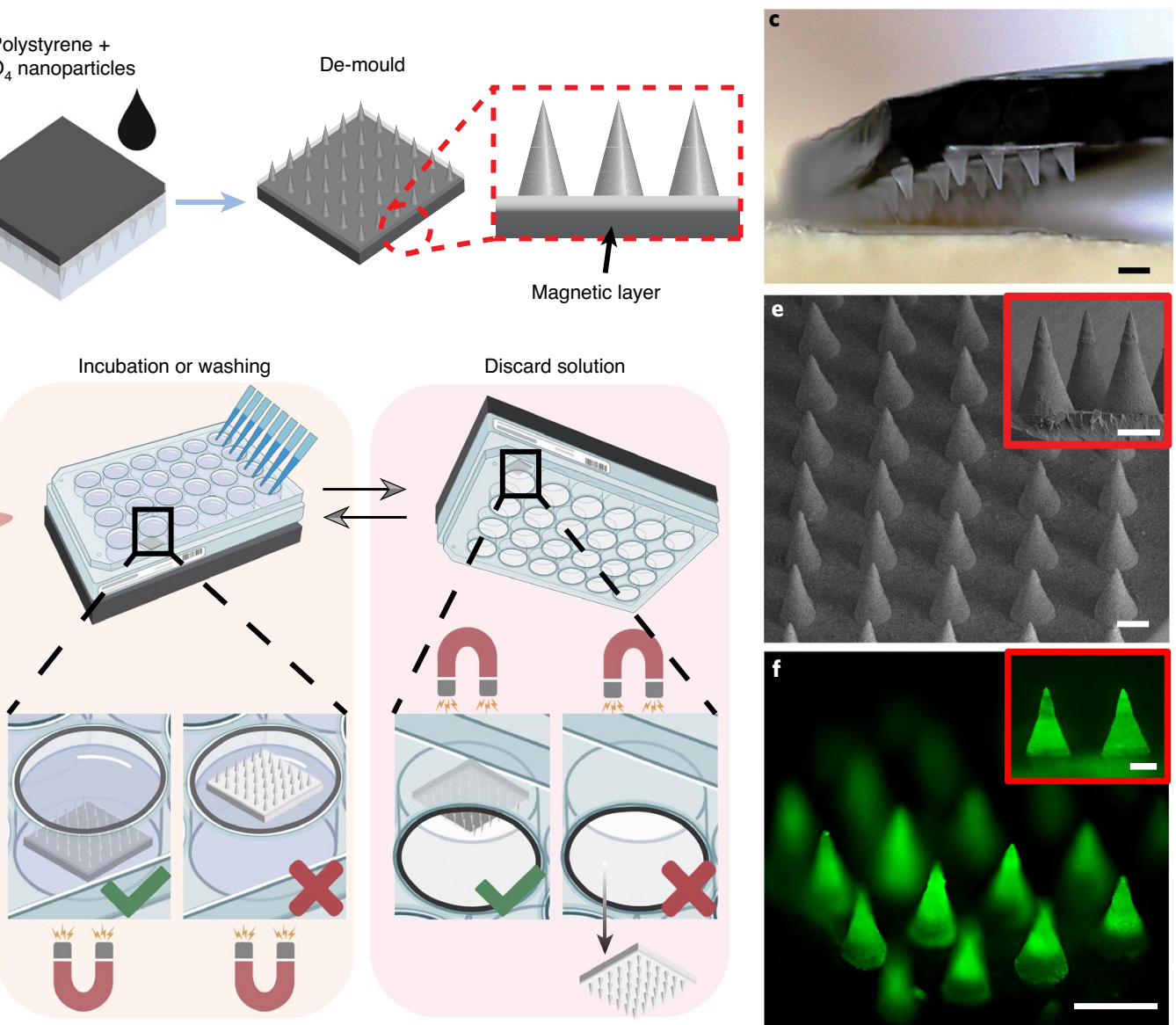

Fig. 1 | Bilayered microneedle fabrication. a, Schematic showing workflow of microneedle-based biodetection involving in situ sampling and on-needle detection of protein biomarkers in ISF. b, Schematic of the fabrication steps for the bilayered microneedle patch. c, Optical image of a microneedle patch with the magnetic backing layer (black) and pristine polystyrene needles (hazy). Scale bar, $500 \mu \mathrm{m}$. d, Schematic depicting the importance of using microneedle patches with embedded magnetic nanoparticles, which ensure that they remain on the plate, facilitating standard immunoassay procedures such as incubation and washing steps, and overcoming low-efficiency patch-by-patch handling. e, SEM image of as-fabricated microneedle patch. Scale bar, $100 \mu \mathrm{m}$. Inset image shows side view of microneedles. Scale bar, $200 \mu \mathrm{m}$. f, Fluorescence microscopy image demonstrating efficient and uniform adsorption of antibodies on polystyrene microneedles. Scale bar, $500 \mu \mathrm{m}$. Inset image shows side view of fluorescence signal on microneedle. Scale bar, $200 \mu \mathrm{m}$.

concentration $\left(2.5 \mathrm{ng} \mathrm{ml}^{-1}\right.$ to $\left.2.5 \mathrm{pg} \mathrm{ml}^{-1}\right)$ were used as standards. Fluorescence signals obtained after applying the plasmonic fluor$800 \mathrm{CW}$ revealed nearly 530 -fold enhancement in the ensemble fluorescence intensity compared with conventional FLISA at the highest IL- 6 concentration tested $\left(2.5 \mathrm{ng} \mathrm{ml}^{-1}\right)$ (Fig. $\left.2 \mathrm{~d}, \mathrm{e}\right)$. The LOD of the IL-6 p-FLISA was found to be around $0.33 \mathrm{pg} \mathrm{ml}^{-1}, 790$-fold lower than that of conventional FLISA $\left(261 \mathrm{pg} \mathrm{ml}^{-1}\right)$ (Fig. $2 \mathrm{~g}, \mathrm{~h}$ ). Scanning electron microscopy (SEM) images revealed the presence of plasmonic fluors on the surface of the microneedle (Fig. $2 \mathrm{f}$, a few plasmonic fluors are highlighted by yellow arrows). Conversely, ELISA performed on the microneedle exhibited weak colorimetric signal and a large standard deviation (Fig. 2i). The optical density corresponding to the highest IL-6 concentration of ELISA on microneedle patch is nearly tenfold lower than the standard ELISA implemented on a microtitre plate, approaching the background noise level (Fig. 2 and Supplementary Fig. 8). The LOD of ELISA on the microneedle patch was measured at around $53 \mathrm{pg} \mathrm{ml}^{-1}, 160$-fold higher than that of $\mathrm{p}$-FLISA $\left(0.33 \mathrm{pg} \mathrm{ml}^{-1}\right)$ (Fig. $\left.2 \mathrm{~h}, \mathrm{i}\right)$. 
a

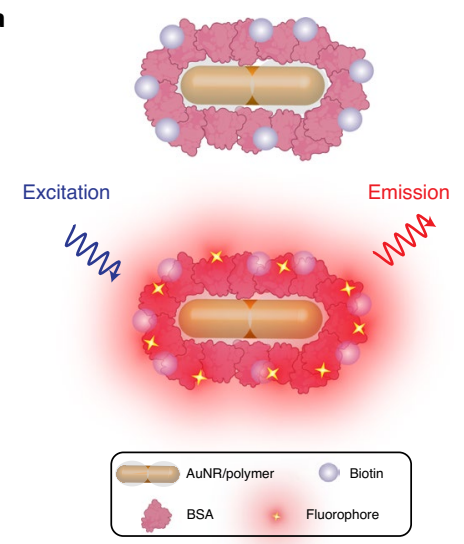

d

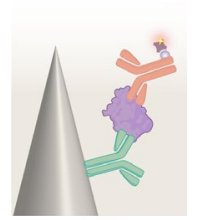

e
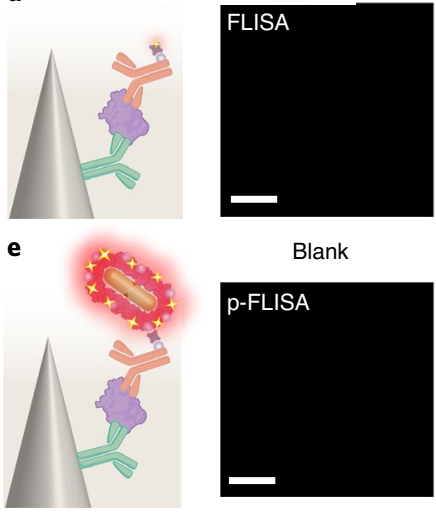

Blank
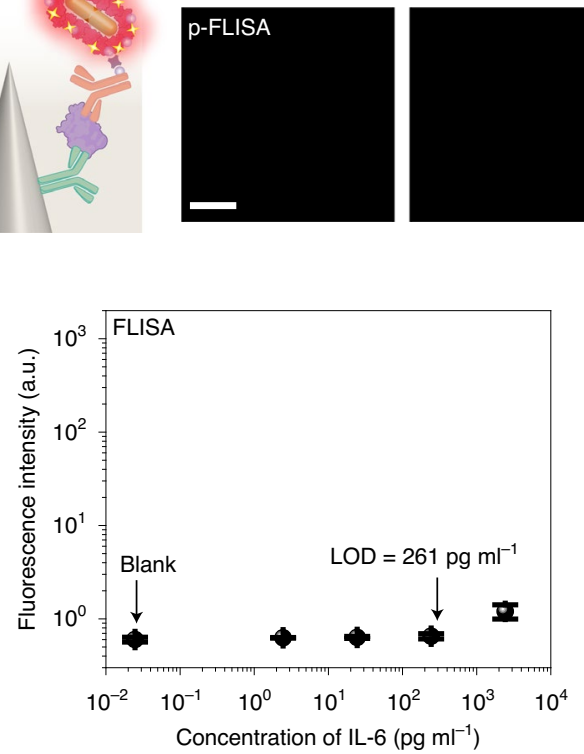

b
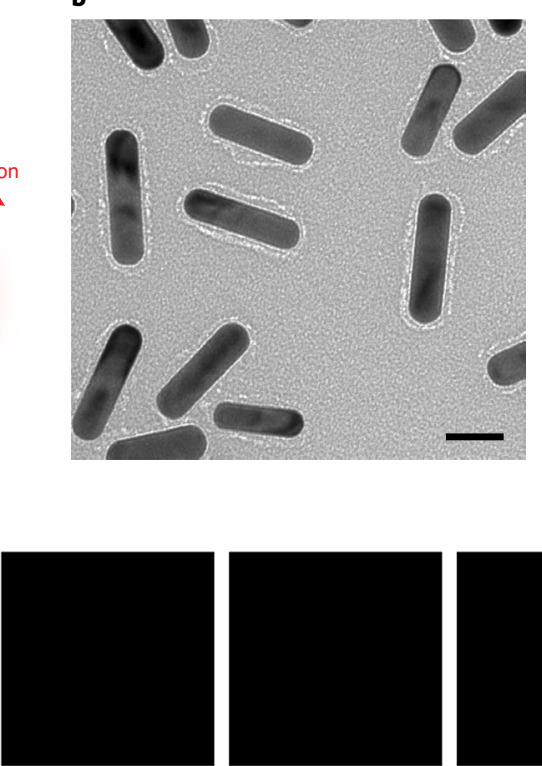

$2.5 \mathrm{pg} \mathrm{ml}^{-1}$

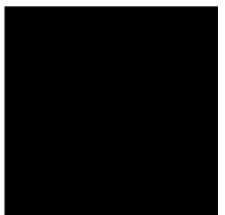

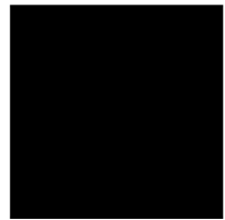

$25 \mathrm{pg} \mathrm{ml}^{-1}$

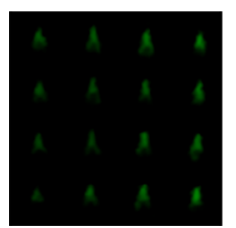

c

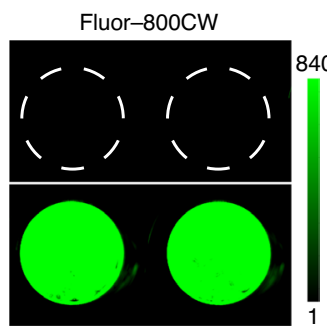

Plasmonic fluor-800CW

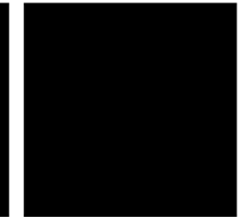

$250 \mathrm{pg} \mathrm{ml}^{-1}$

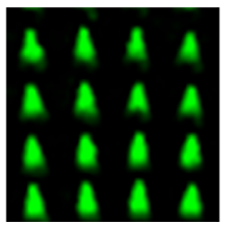

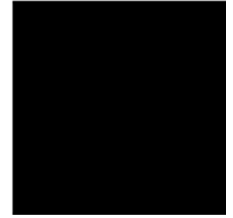

$2,500 \mathrm{pg} \mathrm{ml}^{-1}$

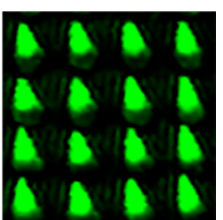

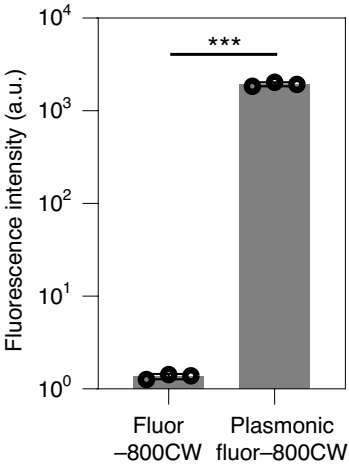

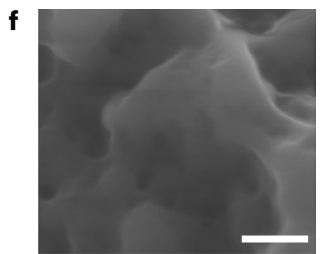

Pristine microneedle

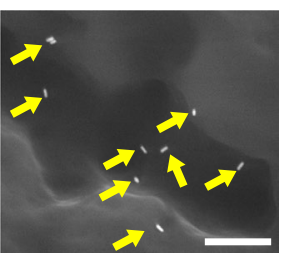

Plasmonic fluor on microneedle
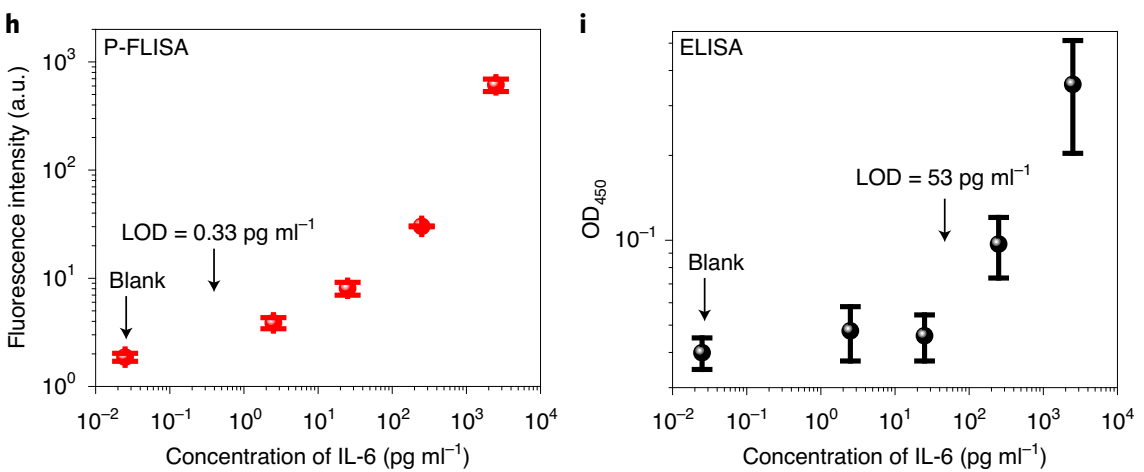

Fig. 2 | p-FLISA on microneedle for ultrasensitive detection. a, Schematic of plasmonic fluor as an ultrabright fluorescence nanolabel. Plasmonic fluor comprises of a plasmonic core (AuNR), a polymer spacer layer, fluorophores, and a universal biorecognition element (biotin), which are assembled using BSA. b, TEM image of plasmonic fluors. Scale bar, $50 \mathrm{~nm}$. c, Fluorescence images and corresponding intensity before and after specific binding of plasmonic fluor through biotin-streptavidin interaction, showing a nearly 1,400-fold increased fluorescence intensity with the plasmonic fluor. Data are mean \pm s.d. $P=0.0004,{ }^{\star \star \star} P<0.001$ by one-tailed unpaired $t$-test with Welch's correction. a.u., arbitrary units. d,e, Fluorescence intensity maps of mouse IL- 6 FLISA (d) and p-FLISA (e) at various analyte concentrations. Scale bars, $500 \mu \mathrm{m}$. f, SEM images showing pristine microneedles (top) and microneedles after being probed with plasmonic fluor (bottom). Plasmonic fluors are highlighted by yellow arrows. Scale bars, $500 \mathrm{~nm}$. $\mathbf{g}$, $\mathbf{h}$, IL- 6 dose-dependent fluorescence

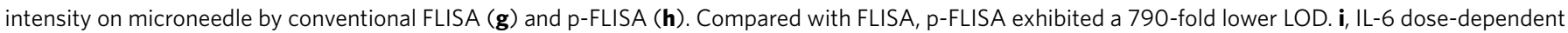
optical density signal at $450 \mathrm{~nm}\left(\mathrm{OD}_{450}\right)$ for ELISA implemented on microneedle patches. Data are mean $\pm \mathrm{s} . \mathrm{d} ., n=3$ repeated tests.

Biophysicochemical properties of microneedle patches. Before deploying the microneedle patches for in vivo transdermal biodetection, we investigated their biophysicochemical properties, such as (1) mechanical strength for penetration of dermal tissue; (2) biocompatibility; and (3) biosafety and potential adverse effects. To determine whether the polystyrene microneedles possess sufficient mechanical strength to penetrate the skin under compression, we performed a micro-compression test on a microneedle patch comprised of an $11 \times 11$ array of microneedles. The microneedle patch tolerated compression force greater than $0.4 \mathrm{~N}$ per microneedle, which is sufficiently high to puncture the skin without causing the microneedles to mechanically yield ${ }^{24}$ (Fig. 3a). The microneedles penetrated the mouse skin, as evidenced by Trypan blue staining (Fig. 3b). Haematoxylin and eosin (H\&E) staining of the extracted mouse skin tissue further confirmed that the microneedles had penetrated the stratum corneum and perforated into the epidermal layer (Fig. 3c and Supplementary Fig. 9). The penetration depth was around $140-170 \mu \mathrm{m}$ (Supplementary Fig. 9). SEM images indicated that the microneedles maintained their conical shape and sharp tips after removal from the mouse skin, further confirming their 
a

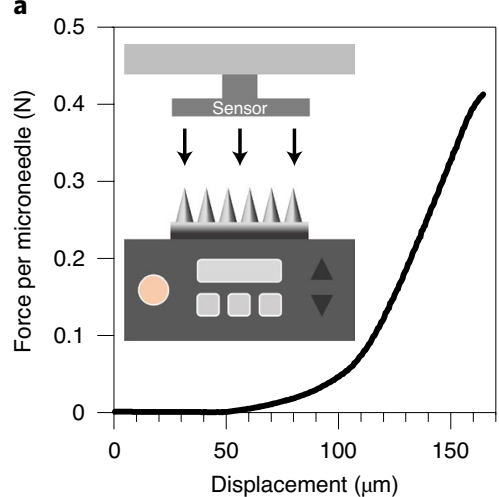

d

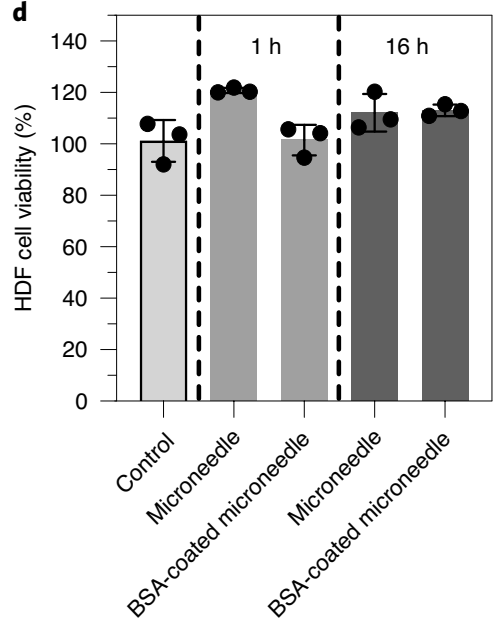

b

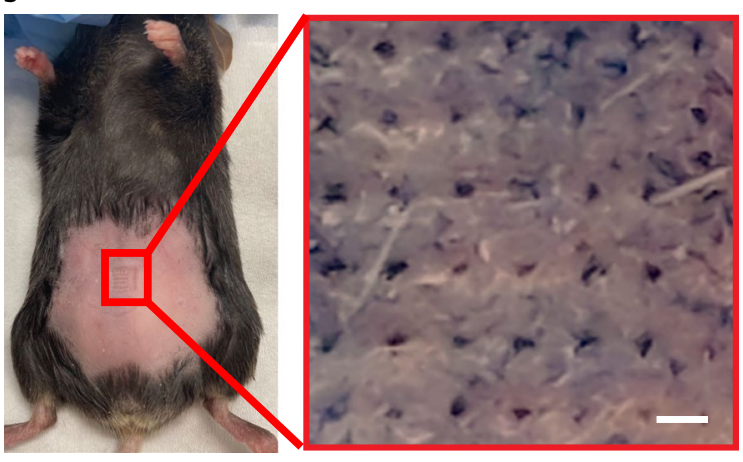

c

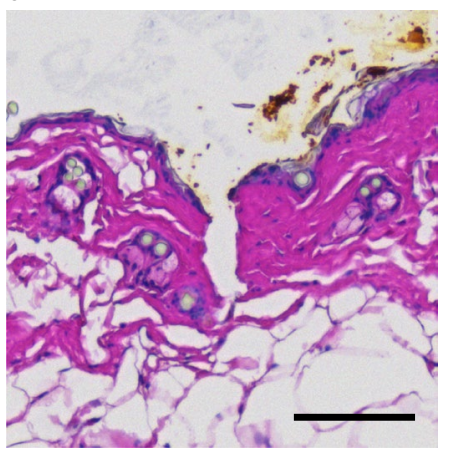

e
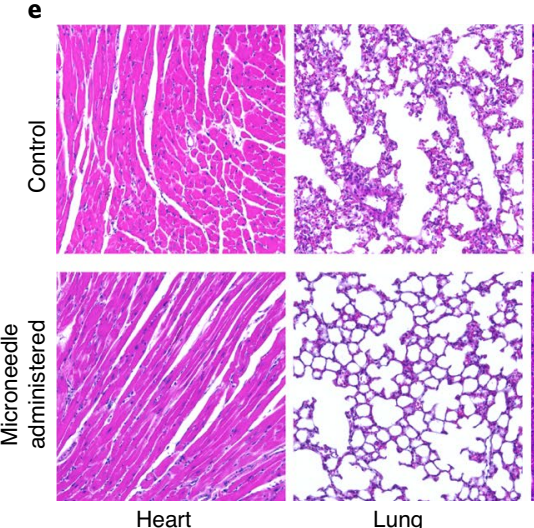

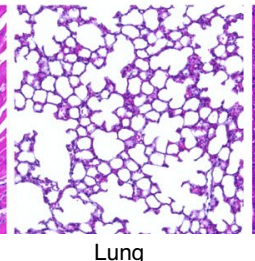

Lung

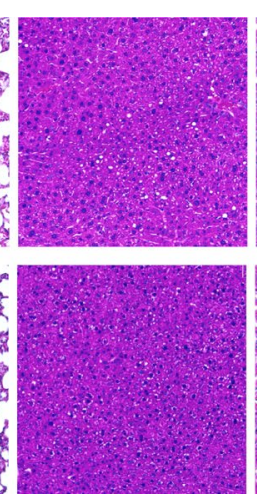

Liver
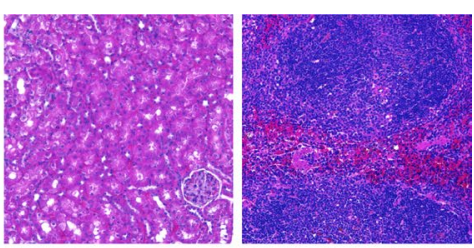
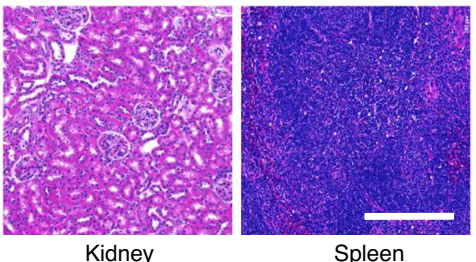

Fig. 3 | Biophysicochemical properties of microneedle patches. a, Mechanical behaviour of the microneedle patches under normal compressive load and schematic of the experimental setup (inset). b, Left: mouse ventral skin administered with a microneedle patch. Right: optical image of trypan blue staining, showing the indents caused by the penetration of microneedles on mouse skin. Scale bar, $500 \mu \mathrm{m}$. c, H\&E-stained section of mouse skin showing the penetration by a single microneedle. Scale bar, $100 \mu \mathrm{m}$. d, Viability of HDFs cultured with medium exposed to pristine and BSA-coated microneedle patches for $1 \mathrm{~h}$ and $16 \mathrm{~h}$. Data are mean \pm s.d., $n=3$ repeated tests. e, H\&E-stained sections of mouse organs with and without administration of microneedle patches, indicating excellent biocompatibility of microneedle patches. Scale bar, $200 \mu \mathrm{m}$.

mechanical integrity during administration (Supplementary Fig. 10). Furthermore, micro-indents on skin caused by the application of the microneedle patch quickly became invisible as the skin recovered to its normal state within $15 \mathrm{~min}$ (Supplementary Fig. 11). The rapid recovery of the skin reaffirms the minimally invasive nature of the procedure compared with conventional techniques such as blood collection.

Considering that the antibodies are immobilized on the microneedles, it is important to understand the stability of these proteins during the penetration of the microneedles into the dermal tissue, residence in the dermal tissue and subsequent withdrawal (Supplementary Fig. 12a). To test this, we coated microneedles with fluorescently labelled BSA (used as a model protein) before application onto mouse skin for different durations $(15 \mathrm{~s}, 10 \mathrm{~min}, 20 \mathrm{~min}$, $30 \mathrm{~min}$ and $60 \mathrm{~min}$ ). Upon withdrawal, microneedles exhibited a loss of around $20 \%$ in fluorescence intensity, indicating that a small fraction of proteins had desorbed from their surface, possibly due to the shear forces between the microneedle surface and the epidermis (Supplementary Fig. 12b). The decrease in the fluorescence intensity (representing the amount of protein lost) did not depend on the residence time of the microneedles in the dermal tissue, indicating minimal loss from desorption or proteolytic degradation of these proteins in the dermal tissue, even for a residence time of $60 \mathrm{~min}$.
To evaluate the biocompatibility of the polystyrene microneedle patches, human dermal fibroblasts (HDFs), one of the major cell types in the dermis layer, were cultured in the presence of microneedles and BSA-coated microneedles (since the in vivo sampling involves microneedles blocked with a dense layer of BSA). Notably, no change in cell viability was observed after either $1 \mathrm{~h}$ or $16 \mathrm{~h}$ of in situ culture with the microneedle patches (Fig. $3 \mathrm{~d}$ ). Systemic toxicity of the microneedle patch was also investigated by H\&E staining. Negligible changes in cell states between the control group and the microneedle-administered group demonstrated the excellent biocompatibility of the microneedle (Fig. 3e). In addition, we measured the endotoxin level of the microneedle patch to investigate the possibility of an unwanted immune response after administration. Microneedles coated with BSA exhibited negligible endotoxin levels compared with the limit set by the US Food \& Drug Administration (20 EU per device) (Supplementary Fig. 13 and Supplementary Table 1). Together, these findings indicate that the biofunctionalized microneedle patch represents a safe approach for in vivo biodetection.

Detection of cocaine-specific antibody in an immunized mouse model. Cocaine overdose and cocaine-use disorder, for which there is currently no FDA-approved medication, remain 
a global medical and social problem ${ }^{25,26}$. While vaccines do not directly address the underlying neurobiological mechanism behind cocaine-use disorder, cocaine vaccines that produce antibodies reduce the rate and quantity of drug entry into the brain and inhibit the psychoactive effects of the drug ${ }^{27}$. Unlike other vaccines that confer prolonged protection, currently designed antidrug vaccines require frequent boosting to maintain the effective antibody levels ${ }^{28,29}$. A recent study revealed that lack of pre-vaccination screening assays that predict the most effective vaccines or subjects amenable to vaccination is the major obstacle for clinical translation ${ }^{30}$. Therefore, technologies that enable rapid and reliable testing of hapten-specific antibody titres in vaccinated subjects may aid rational vaccine design and provide screening tools to predict vaccine clinical efficacy against drugs of abuse. Here, we set out to demonstrate a simple, rapid, and non-invasive method for evaluating the efficacy of the cocaine vaccine using a microneedle patch.

Mice were subcutaneously immunized and boosted with BSAcocaine combined with adjuvants, lipopolysaccharide (LPS) and alum, as depicted in Fig. 4a. This vaccine was expected to result in the generation of two types of santibody: anti-BSA and anti-cocaine. As a standard way to determine antibody titre and immunization efficacy, serum collected on the 7th week was serially diluted and the concentrations of the two antibodies (anti-BSA and anti-cocaine) were tested by the ELISA using BSA and BSA-cocaine as recognition elements, respectively (Fig. 4b,c). Since BSA-cocaine is expected to bind with both types of santibody simultaneously, the difference in the antibody concentrations obtained using BSA-cocaine and BSA represents the concentration of anti-cocaine antibody. Mice with higher anti-BSA-cocaine titre than the anti-BSA titre are considered as vaccine responders (Fig. $4 \mathrm{~d}$ and Supplementary Fig. 14). These responder mice were subjected to microneedle administration to test the ability of this method to detect the presence of anti-cocaine antibody in ISF.

To determine the shortest timescale for effective capture of antibodies, we administered the BSA-coated microneedle patch on the mouse dorsal skin for different durations. We found that $30 \mathrm{~s}$ of microneedle patch administration was sufficient to capture BSA-specific antibodies (Fig. 4e). Longer administration of the microneedle patch did not alter the fluorescence signals corresponding to the antibodies. To assess the site-to-site variations in the measured concentrations, we administered four microneedle patches coated with BSA on the ventral and on the dorsal skin of a responder mouse. The microneedle patches were subsequently probed with anti-mouse IgG and plasmonic fluor ex vivo. We noted very small differences (relative s.d. $<8 \%$ ) in the fluorescence intensity among the four microneedle patches administered on the same side of the mouse. However, the 4 microneedle patches administered on the ventral side exhibited $30-40 \%$ higher fluorescence intensity compared with those on the dorsal side (Fig. 4f). This difference possibly stems from the differences in the thickness of skin and blood flow between ventral and dorsal sides of the mice $^{31,32}$. This observation underscores the importance of accounting for site-to-site variation and consistent administration of the microneedle patches to ensure reliable comparison across different subjects and experiments.

To validate the applicability of the plasmonic fluor-enhanced microneedle patch in detecting cocaine-specific antibodies in a minimally invasive manner, we used five unimmunized mice and five responder mice. Each mouse was administered with two microneedle patches coated with BSA and BSA-cocaine, and the patches were left on the skin for only $30 \mathrm{~s}$. In the five responder mice, we observed high fluorescence signal and significant difference in the fluorescence intensity between the BSA and BSA-cocaine microneedle patches. However, in the case of the microneedle patches from five unimmunized mice, we noted a much lower fluorescence signal and negligible difference between the BSA and BSA-cocaine microneedle patches (Fig. $4 \mathrm{~g}$ ). Together, these results indicate that the plasmonic fluor-enhanced microneedle patch is a promising tool to determine and evaluate the vaccine response efficiency in a simple and non-invasive manner. In contrast to conventional blood drawing, the microneedle method enables frequent and easy monitoring of vaccine efficiency in individuals and can potentially accelerate vaccine development.

Detection and quantification of cytokines in a mouse model of endotoxin-induced shock. Next, we set out to demonstrate the detection of cytokines in dermal ISF in a mouse model of LPS-induced endotoxin shock using the microneedle patch. LPS, a pathogen-associated molecular pattern, triggers innate immunity and induces secretion of multiple pro-inflammatory cytokines (Fig. 5a). Measurement and monitoring of these pro-inflammatory cytokines in blood has been an established method for evaluating the ability of the immune system to mount an innate inflammatory immune response ${ }^{33}$.

BALB/C mice were injected intraperitoneally with LPS $\left(1 \mu \mathrm{gg}^{-1}\right)$ to induce an acute phase response and systemic inflammation. Using functionalized microneedle patches, we measured the longitudinal concentrations of mouse IL-6 in ISF, which is known to increase in serum over $4 \mathrm{~h}$ after LPS administration ${ }^{34}$. Microneedle patches, pre-functionalized with IL-6-capture antibodies, were administered at different time points on mouse ventral skin and left undisturbed for $20 \mathrm{~min}$ (as indicated in Fig. 5b). After removing the microneedle patches from the skin, p-FLISA was performed ex vivo to measure the concentration of IL-6. Fluorescence intensity corresponding to the plasmonic fluors on the microneedle patches exhibited a gradual increase from $1 \mathrm{~h}$ to $4 \mathrm{~h}$ after LPS injection (Fig. $5 \mathrm{c}$ ), whereas no significant change was observed in mice injected with saline (negative control group) (Fig. $5 \mathrm{c}$ and Supplementary Fig. 15). On the basis of the standard curve (obtained using microneedle patches exposed to known concentrations of IL-6), the concentrations of IL-6 in mouse ISF were determined to be equivalent to $2.6 \pm 1.9$, $12.3 \pm 8.6,120.4 \pm 73.4$ and $1271.9 \pm 393.4 \mathrm{pg} \mathrm{m}^{-1}$ at $0,1,2.5$ and $4 \mathrm{~h}$ after LPS injection, respectively (Fig. 5 d). Specificity tests also validated that other cytokines or chemokines, whose levels might also increase with LPS injection, do not interfere with the detection and quantification of IL-6 on microneedles (Supplementary Fig. 17 and Supplementary Table 2). The IL-6 concentration in sera collected $4 \mathrm{~h}$ after LPS or saline injection exhibited good qualitative correlation with that measured in ISF using the microneedle method at the same time point (Fig. 5e). However, the serum IL-6 concentration was found to be nearly 22 -fold higher compared to that in the ISF. The absolute concentration of the protein biomarkers in ISF was lower than the concentration in blood, which can partly be ascribed to the difference between microneedle-based analyte sampling method and solution-based standard curve, as well as inherent variation of proteins in body fluids ${ }^{4}$. The p-FLISA standard curve, which is used for estimating the concentration of the analyte, was obtained by exposing the microneedle patches to known concentrations of IL-6 in standard dilution buffer. By contrast, microneedle-based ISF sampling occurs in a dense tissue matrix, resulting in slower diffusion kinetics and, consequently, a lower apparent concentration of the analyte. Nevertheless, the analyte concentration determined using the microneedle-based method also exhibited excellent qualitative agreement with the measured concentrations in serum samples (Fig. 5e and Supplementary Fig. 16).

Frequent and timely measurement of protein biomarkers is critical for disease monitoring and diagnostics in both biomedical research and clinical applications. Unfortunately, conventional longitudinal measurements require frequent blood draws in a short period, which may cause iatrogenic anaemia and increase morbidity of patients. Moreover, it is often impossible to repeatedly draw 
a

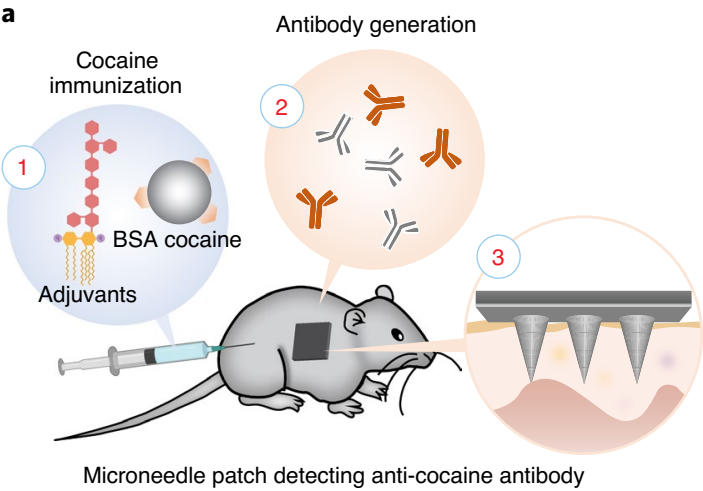

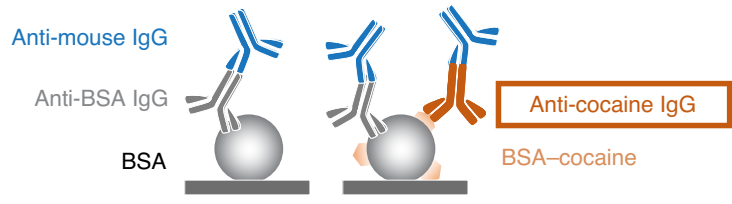

On microneedle patches or microtitre plates

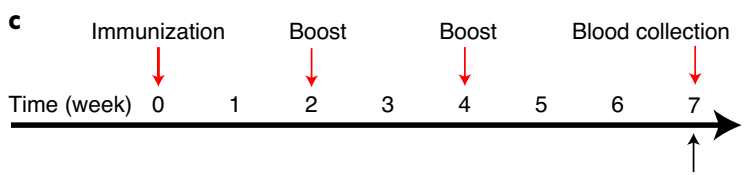

d

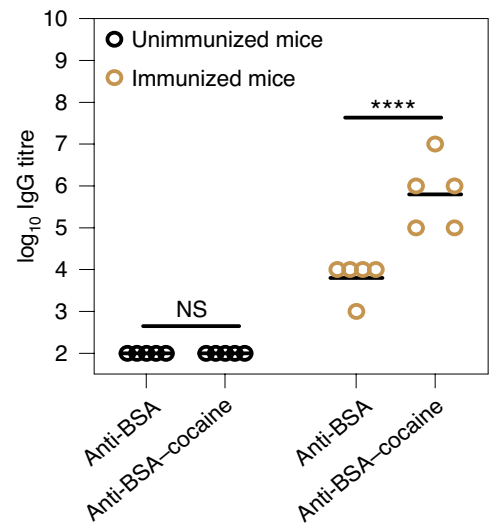

e

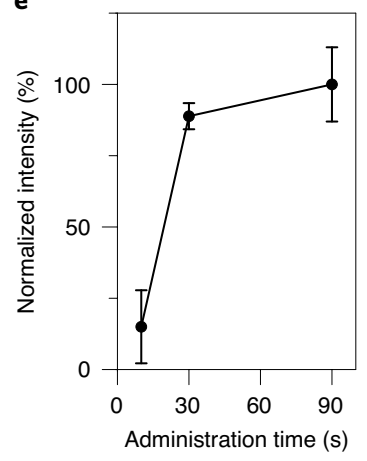

Microneedle administration

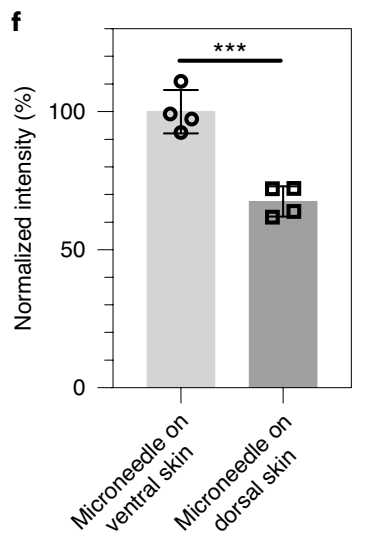

g

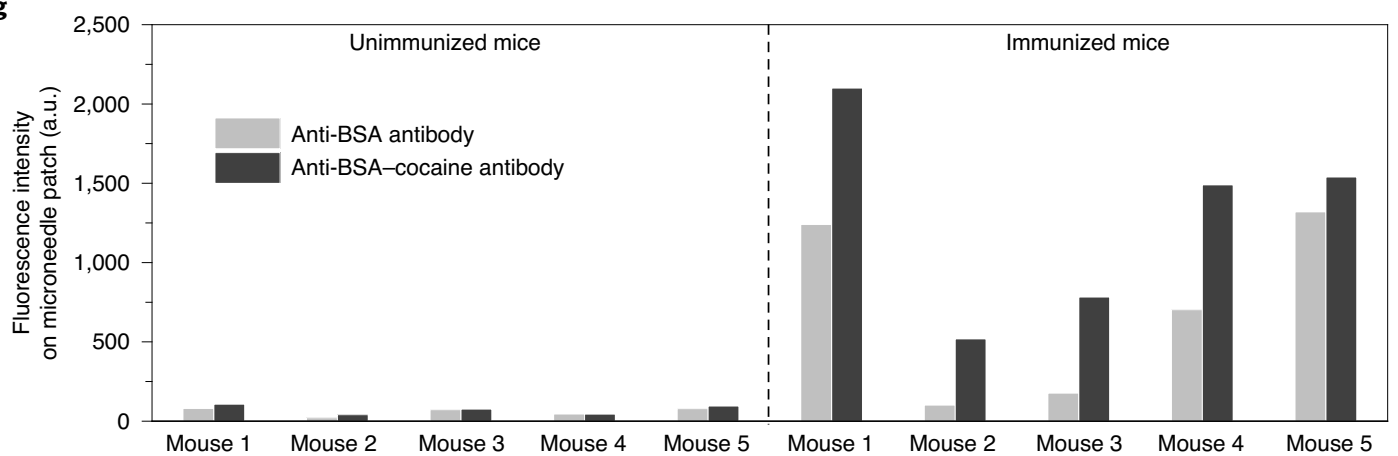

Fig. 4 | Minimally invasive detection of cocaine-specific antibodies in an immunized mouse model. a, Schematic showing the working principle of cocaine immunization, generation of BSA and cocaine-specific antibodies, and administration of the microneedle patch for the detection of specific antibodies in ISF. b, Schematic demonstrating the principle of anti-cocaine antibody detection. Two types of antibody are generated after cocaine immunization: anti-BSA and anti-cocaine. Since BSA-cocaine binds simultaneously to both types of antibody, the difference between the antibody concentrations determined using BSA-cocaine- and BSA-coated plates represents the concentration of anti-cocaine antibody. c, Workflow of immunization, blood draw and microneedle administration on mice. $\mathbf{d}, \log _{10}$ titre of anti-BSA and anti-BSA-cocaine in mouse serum from immunized and unimmunized groups, tested by gold standard ELISA. $n=5$ mice per group. Among the unimmunized group, the data were not significantly different between anti-BSA and anti-BSA-cocaine ( $P>0.9999$ by one-way analysis of variance (ANOVA) with Tukey's multiple-comparison test). Among the immunized group, data were statistically significant between anti-BSA and anti-BSA-cocaine ( ${ }^{\star \star \star \star} P<0.0001$ by one-way ANOVA with Tukey's multiple-comparison test). NS, not significant. e, Plot showing normalized fluorescence intensity (with respect to highest intensity) on microneedle patches, which were administered on ventral mouse skin and left for 15,30 and 90 s. Error bars represent s.d. $n=3$ repeated tests. f, Plot showing normalized fluorescence intensity on microneedle patches administered on the ventral and dorsal skin of a mouse. Error bars represent s.d. $n=4$ repeated tests. Data statistically significant $P$ value $=0.0004,{ }^{\star \star \star} P<0.001$ by one-tailed unpaired $t$-test with Welch's correction. $\mathbf{g}$, Plot showing the fluorescence intensity obtained from the microneedle patch with BSA or BSA-cocaine coating from both immunized and unimmunized groups.

blood from small experimental animals, which can result in their death. The minimally invasive microneedle method represents a transformative approach to perform frequent, sensitive and accurate measurements of protein biomarkers in a longitudinal manner in the same mouse.
As opposed to in vitro diagnostics that involve sample acquisition (such as blood or urine) at a specific time point along the disease progression and analysis at a later point in time, in vivo diagnostics involving capture of target analytes from a dynamically varying matrix (for example, dermal ISF in the present case) 


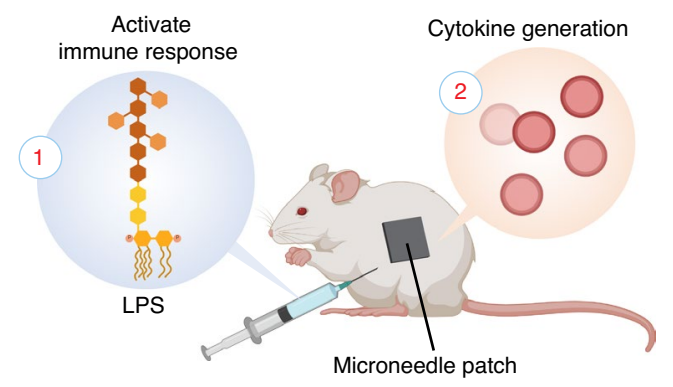

b

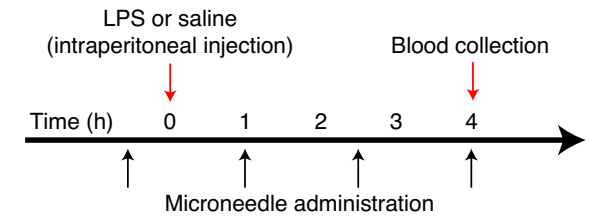

c

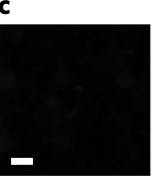

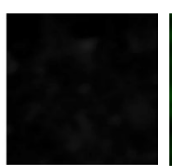

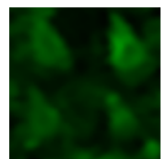

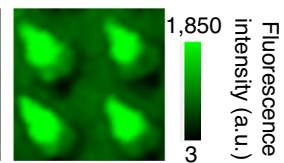

d

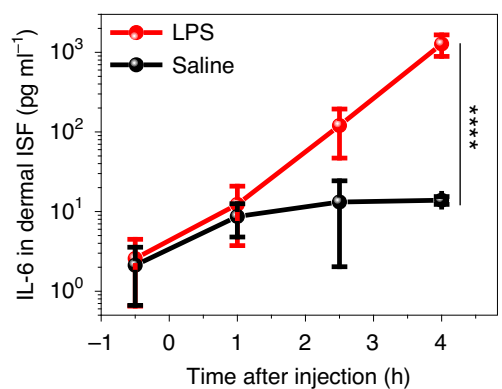

e

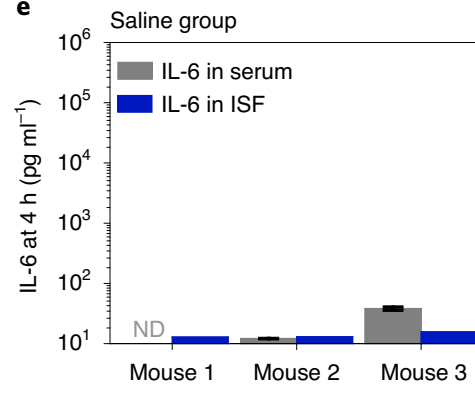

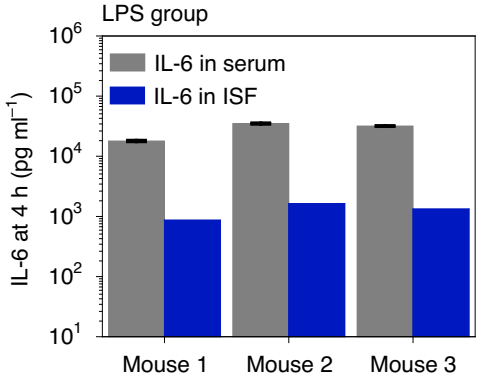

Fig. 5 | Longitudinal monitoring and quantification of cytokines in a mouse model of endotoxin shock. a, Schematic showing the working principle of immune response induced by LPS administration, cytokine generation and administration of the microneedle patch for longitudinal detection. b. Time line of endotoxic shock (LPS injection), longitudinal microneedle administration on mice and blood collection (for validation of the microneedle test). c, Fluorescence map representing mouse IL-6 captured on the microneedles before and at 1, 2.5 and $4 \mathrm{~h}$ after LPS injection. Scale bar, $200 \mu \mathrm{m}$. d, Plot depicting concentrations of mouse IL-6 in ISF before and at 1, 2.5 and $4 \mathrm{~h}$ after LPS or saline injection, measured using microneedles. $n=3$ in each group. ${ }^{\star \star \star} P<0.0001$ by two-way ANOVA with Sidak's multiple-comparison test. e, Comparison of concentrations of IL- 6 measured in serum and ISF in the saline group (left) and the LPS group (right) ( $n=3$ per group) 4 h after LPS injection. IL- 6 in dermal ISF determined by plasmonic fluor-enhanced microneedle patch (blue) exhibited good qualitative correlation with that in serum tested by conventional ELISA (grey). Data are mean \pm s.d. Serum results are technical repeats from one sample. ND, not detected.

is inherently a non-equilibrium condition. This is particularly true for the cases in which the target analyte concentration varies within the sampling timescales, for example, IL-6 level in LPS-stimulated mice. In such cases, the concentration determined using microneedle patches represents a time-average concentration of the analyte in ISF over the sampling period. From a diagnostic translation standpoint, this time-averaged concentration can be standardized by setting rigorous guidelines for microneedle administration (for example, administration time, location) and ex vivo analysis (for example, standard curve conditions).

Detection and quantification of endogenous matricellular protein in periosteum. The quantification of endogenous biomarkers at specific sites or inner tissues of interest is highly desirable for biomedical and clinical research. Conventional biofluids, such as blood, are not able to reflect local concentrations of biomarkers. Furthermore, such local detection and monitoring of relevant biomolecules remains challenging due to the difficulty in collecting an adequate amount of biofluid in a minimally invasive manner. While invasive procedures are sometimes inevitable for detection of protein biomarkers in inner tissues or organs, the microneedle-assisted technique will be able to minimize the damage to the target sites and allow repeated sampling. Here we hypothesized that the microneedle patch would efficiently sample and measure protein analytes at specific tissue or membrane locations with high sensitivity and specificity in a minimally destructive manner (Fig. 6a).

The periosteum is a fibro-cellular membrane, which covers the outer surface of bones and has key roles in bone growth, fracture healing and skeletal regeneration ${ }^{35,36}$. In rodents and humans, the average thickness of the periosteum is $40 \mu \mathrm{m}$ and $100 \mu \mathrm{m}$ for tibia and femur, respectively ${ }^{37,38}$. In our study, the average periosteal thickness in the mouse calvaria was $29 \pm 15 \mu \mathrm{m}$ (Fig. 6b). The extracellular matrix of the periosteum contains a high local concentration of the matricellular protein periostin-encoded by the Postn gene-which has important roles in bone regeneration and bone tumour metastasis ${ }^{39,40}$. In addition, a circulating isoform of periostin has been identified as a potential biomarker of bone density loss, osteoporosis, tumour metastasis and airway disease ${ }^{41,42}$. We set out to test the applicability of our microneedle patch for the detection and quantification of periostin in the calvarial periosteum and in dermal ISF.

We used Postn ${ }^{K O}$ and WT mice as experimental and control groups, respectively. WT mice demonstrated high expression of periostin protein in the calvarial periosteum by immunohistochemistry, which was absent in Postn ${ }^{K O}$ mice (Fig. 6b). To detect periostin, microneedles were pre-functionalized with anti-periostin antibodies and administered to the calvarial periosteum layer for 10 min (Fig. 6c). Simultaneously, another pre-functionalized patch was administered on the dorsal skin of the same mouse for $10 \mathrm{~min}$. Subsequently, p-FLISA assay was performed ex vivo to measure the local concentrations of periostin. Fluorescence intensity corresponding to the plasmonic fluors on the microneedle patches (both on periosteum and dorsal skin) exhibited a strong signal from WT mice, whereas no significant signal was observed in Postn ${ }^{K O}$ mice (Fig. 6d). On the basis of the standard curve, concentrations of periostin in periosteum and skin were around $2,000 \mathrm{pg} \mathrm{ml}^{-1}$ in WT mice, compared with a negligible amount in Post ${ }^{K O}$ mice.

Compared to conventional methods, the plasmonic fluor-enhanced microneedle method did not require extraction or isolation of periosteum during analysis, indicating that multi-round 


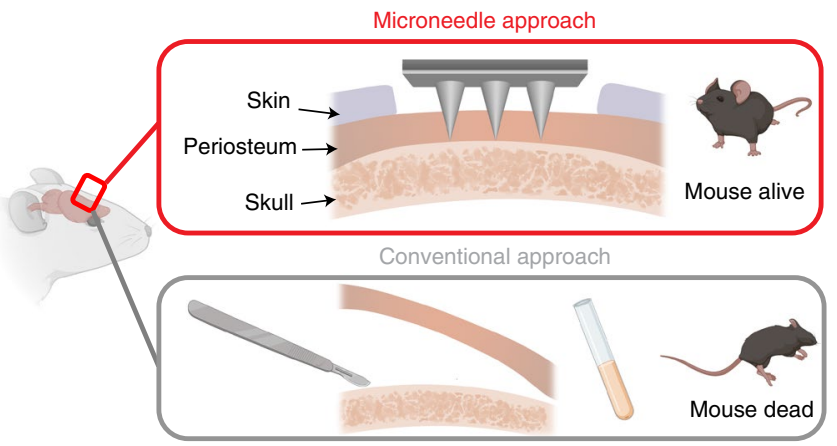

b

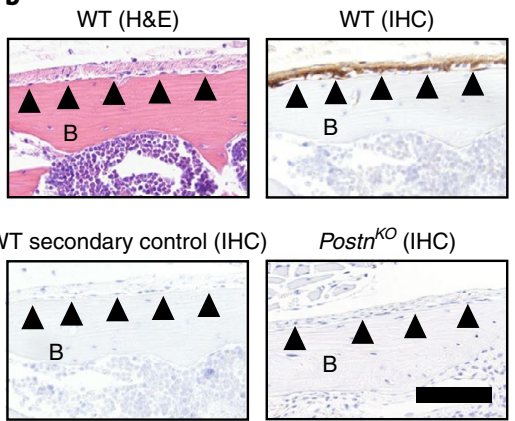

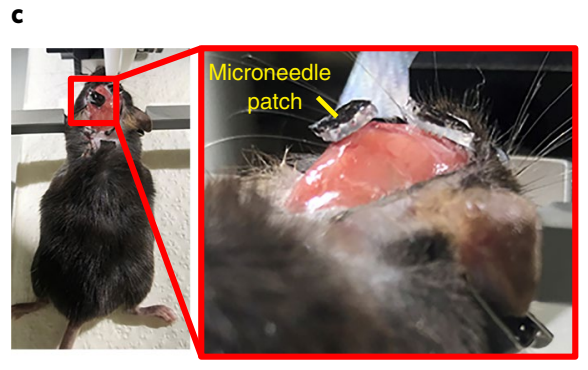

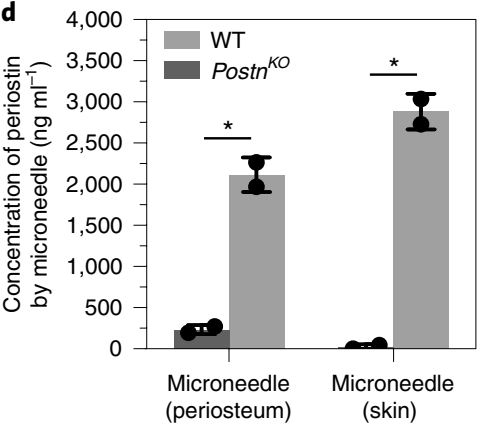

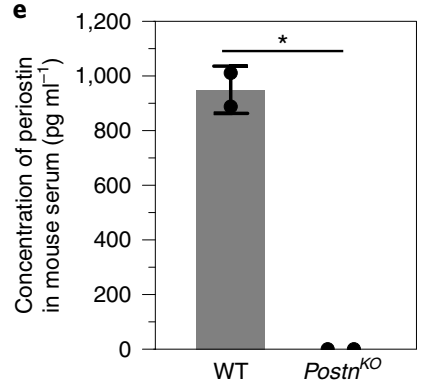

Fig. 6 | Detection of periostin in periosteum by microneedle patch. Endogenous matricellular periostin was detected by a microneedle patch in calvarial periosteum and skin of the WT mice but not the Post ${ }^{K O}$ mice. a, Schematic showing the microneedle and conventional approaches to measuring endogenous biomarkers in calvarial periosteum. Microneedle detection is minimally destructive, and mice can recover after testing; standard analysis requires scraping of the skull and isolation of periosteum, which usually requires euthanasia. b, H\&E staining and immunohistochemistry (IHC), showing a high local concentration of periostin. The calvaria of WT and Post ${ }^{K O}$ mice were immunostained with antibodies against periostin (brown).The periosteum (top, arrowheads) of WT mice exhibits strong expression of periostin, whereas staining was absent in sections from WT mice processed without primary antibody (secondary control) and in sections of calvaria from Postn ${ }^{K O}$ mice. Across the surface of the skull, the average thickness of the calvarial periosteum was $29 \pm 15 \mu \mathrm{m}$. B represents the position of bone. c, Optical images showing the administration of the microneedle patch on mouse calvarial periosteum. The head of the mouse was stabilized on a stereotaxic instrument. d, Concentration of periostin in the skin and periosteum of the WT and Post ${ }^{K O}$ mice determined with microneedle patches. Both the periosteum and skin of WT mice $(n=2)$ demonstrate high concentrations of periostin, whereas Post $n^{K O}$ mice $(n=2)$ show negligible amounts. Data are mean \pm s.d. $P=0.0206$ for microneedle patch on periosteum; $P=0.0153$ for microneedle patch on skin; ${ }^{\star} P<0.1$ by one-tailed unpaired $t$-test with Welch's correction. e, Concentration of periostin in serum of the WT and $P$ ostn ${ }^{K O}$ mice. Serum of WT mice shows high concentrations of periostin, whereas $P$ ostn ${ }^{K O}$ mice have a negligible amount. Data are mean $\pm \mathrm{s}$.d. $P=0.0205$; ${ }^{\star} P<0.1$ by one-tailed unpaired t-test with Welch's correction.

testing and long-term monitoring is achievable (Fig. 6a). Notably, the concentration of periostin in periosteum and skin measured by microneedle is nearly 450 -fold and 330 -fold lower, respectively, than the concentration in mouse serum (around $950 \mathrm{ng} \mathrm{ml}^{-1}$ ) (Fig. 6e). This phenomenon is possibly caused by binding and retention of periostin within the extracellular matrix, reducing the amount of available perostin that can be captured by the microneedle. Since the sampling area for skin and periosteum is different, absolute comparison of the values between these two tissues is precluded. It is also worth mentioning that blood periostin represents a systemic level of periostin while local periostin plays important roles in stem cell recruitment. Understanding its specific level is critical especially when there is a change introduced to the local environment of periosteum or bone. For example, if fracture occurred on the bone and the regeneration process has started, the local level of periostin at defined locations around this fracture can be revealed without substantially disturbing the regeneration process. In this way, it is easier to track the progress of bone regeneration and better understand how periostin contributes to this process by looking at changes in local periostin levels. The same concept could be applied in diverse fields to analyse desired target biomarkers and understand disease progression. In general, these results indicate that the microneedle patch can be used to evaluate the amount of an endogenous matricellular protein biomarker at specific tissue locations in a minimally invasive manner.

\section{Discussion}

In summary, we have designed a minimally invasive, ultrasensitive and quantitative biodetection technology based on a bilayered microneedle patch with plasmonic-enhanced fluoroimmunoassay. Using a series of mouse models, we demonstrate that this technology enables simple and timely detection of biomarkers of interest, including longitudinal monitoring of inflammatory immune responses, evaluation of vaccine efficiency in a minimally invasive manner, as well as quantification of localized changes in protein content within the tissue microenvironment. While largely retaining or enhancing the sensitivity and convenient workflow of conventional immunoassays, the plasmonic-enhanced microneedle method overcomes the need for tedious sample collection (for example, blood draw or ISF extraction), making it highly attractive for clinical diagnostics. Although we focused on the detection of antibodies, cytokines and matricellular proteins in this work, the biodetection method may be broadly applicable to a wide range of other diseases for which rapid and timely diagnosis and personalized treatment are effective interventions. For example, the diagnosis of myocardial infarction could be expedited by applying the microneedle patch (specific to cardiac troponin) in the ambulance as the patient is being moved to the emergency room after experiencing chest pain. Moreover, this technology could enable at-home sampling and centralized detection of biomarker levels through self-administration of microneedle patch, 
which can ultimately enable timely monitoring of therapy, such as certain antibiotic treatments, chemotherapy or checkpoint inhibitor immunotherapy. Therefore, this sensing technique can potentially be employed in point-of-care and resource-limited settings to facilitate rapid disease diagnosis and efficient therapeutic intervention in a more patient-friendly manner.

We note that various protein biomarker levels in the ISF are not well characterized and understood, which is an important bottleneck in the clinical translation of this technology ${ }^{4}$. Extensive efforts need to be dedicated to understanding the biomarker levels in ISF, their correlation with blood and, more importantly, the associated lag time correlated with blood, before this technology can be employed in clinical diagnostics. The technology demonstrated here serves as an efficient tool for biomedical and clinical researchers to fill this knowledge gap and propel the technology towards clinical applications.

\section{Methods}

Animals. All procedures have been approved by the Institutional Animal Care and Use Committee (IACUC) at Washington University in St Louis. Mice were housed in the housing facility at a constant temperature $\left(21-23^{\circ} \mathrm{C}\right)$ and humidity $(45-50 \%)$ on a $12 \mathrm{~h}$ light-dark cycle (lights on 07:00-19:00 h), with food and water available ad libitum throughout the studies.

Synthesis of magnetic nanoparticles. $\mathrm{Fe}_{3} \mathrm{O}_{4}$ nanoparticles were synthesized via previously reported polyol synthesis method ${ }^{43}$. In brief, $0.15 \mathrm{~g}$ of iron (III) chloride hexahydrate (Sigma Aldrich, 236489) was dissolved in a mixture of $3 \mathrm{ml}$ ethylene glycol (Sigma Aldrich, 102466) and $1 \mathrm{ml}$ ethanolamine (Sigma Aldrich, 398136) to form a stable light-brown solution. After $30 \mathrm{~min}, 167.5 \mathrm{mg}$ of polyethylene glycol (Sigma Aldrich, P3640) and $663.6 \mathrm{mg}$ of sodium acetate trihydrate (Sigma Aldrich, S8625) was added under vigorous stirring. Subsequently, the solution was transferred to a Teflon-lined stainless-steel autoclave and heated to $200^{\circ} \mathrm{C}$ for $8 \mathrm{~h}$. The $\mathrm{Fe}_{3} \mathrm{O}_{4}$ nanoparticles were collected and washed three times with ethanol and water, and re-dispersed in dichloromethane for further use.

Fabrication of microneedles. Microneedles were prepared using silicone moulds with conical holes (Blueacre Technology). Each microneedle was $600 \mu \mathrm{m}$ in length with a diameter of $300 \mu \mathrm{m}$ at the base. The centre-to-centre spacing between the microneedles was $600 \mu \mathrm{m}$. Polystyrene-dichloromethane solution $(25 \% \mathrm{w} / \mathrm{v})$ was deposited on the silicone mould and left under vacuum at the bottom to create a negative pressure, followed by drying at room temperature for at least $4 \mathrm{~h}$, allowing the evaporation of the solvent and the formation of the polystyrene microneedle. $\mathrm{Fe}_{3} \mathrm{O}_{4}$ nanoparticles dispersed in polystyrene-dichloromethane solution (final concentration: $15 \% \mathrm{w} / \mathrm{v}$ of polystyrene and $8 \mathrm{mg} \mathrm{ml}^{-1} \mathrm{Fe}_{3} \mathrm{O}_{4}$ nanoparticles in dichloromethane) were deposited on the back of the polystyrene microneedle patch, followed by vacuum. After drying at room temperature for $4 \mathrm{~h}$, the microneedle patch was carefully separated from the mould and tailored into small square pieces. The preparation and storage of microneedle patches were performed in sterile conditions.

Synthesis of plasmonic fluor. Synthesis of plasmonic fluor was performed according to a procedure described previously ${ }^{22}$.

Synthesis of AuNR. The wavelength of gold nanorods can be easily tuned to couple with dye molecules to achieve the best enhancement factor ${ }^{44}$. To prepare plasmonic fluor-800CW, AuNR-760 (localized surface-plasmon resonance wavelength $\sim 760 \mathrm{~nm}$ ) was prepared by the seed-mediated method ${ }^{45,46}$. To prepare seed solution, $0.6 \mathrm{ml}$ of $10 \mathrm{mM}$ ice-cold $\mathrm{NaBH}_{4}$ solution (Sigma Aldrich, 71321) was added into a solution containing $9.75 \mathrm{ml} 0.1 \mathrm{M}$ hexadecyltrimethylammonium bromide (CTAB) (Sigma Aldrich, H5882) and $0.25 \mathrm{ml} 10 \mathrm{mM} \mathrm{HAuCl}_{4}$ (Sigma Aldrich, 520918) under vigorous stirring at room temperature for $10 \mathrm{~min}$. The solution changed from yellow to brown, indicating the formation of Au seed. To synthesize gold nanorods, the growth solution was prepared by the sequential addition of $2 \mathrm{ml} 0.01 \mathrm{M} \mathrm{HAuCl}_{4}$ aqueous solution, $38 \mathrm{ml} \mathrm{0.1} \mathrm{M} \mathrm{CTAB,} 0.55 \mathrm{ml} 0.01 \mathrm{M} \mathrm{AgNO}_{3}$ (Sigma Aldrich, 20439 0), $0.8 \mathrm{ml} 1 \mathrm{M} \mathrm{HCI}$ (Sigma Aldrich, H9892) and $0.22 \mathrm{ml}$ $0.1 \mathrm{M}$ ascorbic acid (Sigma Aldrich, A92902) followed by gentle homogenization. Subsequently, $5 \mu \mathrm{l}$ of the seed solution was added into the growth solution and left undisturbed in dark for $24 \mathrm{~h}$. AuNR solution was collected by centrifugation at $6,000 \mathrm{rpm}$ for $40 \mathrm{~min}$ to remove the supernatant. AuNR was then re-dispersed into nanopure water for further use.

Conjugation procedures. BSA was first conjugated with biotin and $800 \mathrm{CW}$ sequentially through EDC-NHS chemistry. Specifically, 2 mg NHS-PEG4-biotin (Thermo Scientific, 21329) was added to $2.2 \mathrm{ml}$ of $5 \mathrm{mg} \mathrm{ml}^{-1}$ BSA (Sigma Aldrich, A7030) in $1 \times \mathrm{PBS}$ and incubated at room temperature for $1 \mathrm{~h}$. BSA-biotin conjugation was purified by a desalting column (Thermo Scientific, 21329, 7,000 MWCO).
Next, 800CW was conjugated to BSA-biotin. One-hundred microlitres of $1 \mathrm{M}$ $\mathrm{K}_{2} \mathrm{HPO}_{4}$ buffer $\mathrm{pH} 9$ was added into $1 \mathrm{ml}$ purified BSA-biotin solution to raise the pH. Next, $25 \mu \mathrm{l} \mathrm{of} 4 \mathrm{mg} \mathrm{ml}^{-1}$ NHS-800CW (LI-COR, 929-70020) was added to the mixture, and the solution was incubated at room temperature for $2.5 \mathrm{~h}$. BSA-biotin-800CW was purified using a Zeba desalting column pre-equilibrated with nanopure water.

Synthesis of plasmonic fluor. To prepare plasmonic fluor-800CW, AuNR (wavelength around $760 \mathrm{~nm}$ ) was employed as the nanoantenna. One microlitre (3-mercaptopropyl)trimethoxysilane (MPTMS) (Sigma Aldrich, 175617) was added to $1 \mathrm{ml}$ AuNR (extinction $~ 2$ ) and the mixture was shaken on rocking bed for $1 \mathrm{~h}$. Subsequently, MPTMS-modified AuNR was collected by centrifugation at $6,000 \mathrm{rpm}$ for $10 \mathrm{~min}$ and was further mixed with $2 \mu \mathrm{l}$ APTMS (Sigma Aldrich, 281778) and $2 \mu$ l trimethoxy(propyl)silane (TMPS) (Sigma Aldrich, 662275) to form the polymer spacer layer. Finally, AuNR-polymer solution was collected by two centrifugations at $6,000 \mathrm{rpm}$ for $10 \mathrm{~min}$ to remove the free monomer and concentrated into a final volume of $10 \mu \mathrm{l}$.

Next, BSA-biotin-800CW conjugate was coated around AuNR-polymer modified using a previously reported method ${ }^{47}$. Specifically, $\mathrm{pH}$ of $100 \mu \mathrm{l} 4 \mathrm{mg} \mathrm{ml}^{-1}$ BSA-biotin-800CW was first lowered by adding $1 \mu \mathrm{l}$ of $20 \mathrm{mg} \mathrm{ml}^{-1}$ citric acid (Alfa Aesar, 36664). Subsequently, concentrated AuNR-polymer solution was added into BSA-biotin-800CW solution and sonicated for $20 \mathrm{~min}$ under dark condition. The coated nanostructures were then collected by centrifugation at $5,000 \mathrm{rpm}$ for $5 \mathrm{~min}$ and subsequently incubated with $0.5 \mathrm{ml}$ of $0.4 \mathrm{mg} \mathrm{ml}^{-1}$ BSA-biotin-800CW (pH 10) for $3 \mathrm{~d}$ at $4{ }^{\circ} \mathrm{C}$. Finally, the nanostructures were washed 4 times using alkaline nanopure water $(\mathrm{pH} 10)$ by centrifugation at $6,000 \mathrm{rpm}$ and re-dispersed in $1 \%$ BSA in $1 \times$ PBS solution for further use.

Fluorescence enhancement using plasmonic fluor. The test procedure is schematically illustrated in Supplementary Fig. 7. Specifically, BSA-biotin was first immobilized on a 96-well plate by incubating the well with $50 \mathrm{ng} \mathrm{ml}^{-1}$ BSAbiotin in $1 \times$ PBS at room temperature for $15 \mathrm{~min}$. The plate was washed by three times using PBST ( $0.05 \%$ Tween 20 in $1 \times$ PBS) and then blocked using Odyssey Blocking Buffer (PBS) (LI-COR, 927-40100). Streptavidin-800CW $\left(1 \mu \mathrm{g} \mathrm{ml}^{-1}\right)$ was subsequently added and incubated for $10 \mathrm{~min}$. Next, the plate was washed three times using PBST and then incubated with $\sim 76 \mathrm{pM}$ plasmonic fluor-800CW (in $1 \%$ BSA). Finally, after washing, $200 \mu \mathrm{l}$ of PBST was added to each well and the fluorescence signals before and after the addition of plasmonic fluor were recorded using a LI-COR CLX fluorescence imager with the following scanning parameters: laser power $\sim$ L2; resolution $\sim 169 \mu \mathrm{m}$; channel: 800 ; height: $4 \mathrm{~mm}$.

Material characterization. TEM images were obtained using a JEOL JEM$2100 \mathrm{~F}$ field emission instrument. A drop of aqueous solution was dried on a carbon-coated grid, which had been made hydrophilic by glow discharge. SEM images were obtained using a FEI Nova 2300 field-emission SEM at an acceleration voltage of $10 \mathrm{kV}$. The extinction spectra of plasmonic nanostructures were obtained using a Shimadzu UV-1800 spectrophotometer. Fluorescence mappings were recorded using LI-COR Odyssey CLx imaging system. The X-ray diffraction (XRD) patterns of the $\mathrm{Fe}_{3} \mathrm{O}_{4}$ nanoparticles were obtained using a Bruker D8-Advance X-ray powder diffractometer using $\mathrm{Cu}$ K $\alpha$ radiation $(\lambda=1.5406 \AA)$ over the $2 \theta$ range $10^{\circ}-90^{\circ}$.

Mechanical test. The mechanical properties of the microneedle patch were measured with a displacement-force test station (Instron 5583 Electro-mechanical Universal Testing Machine) (Fig. 3a). A microneedle patch was attached to a rigid platform with microneedles facing up. The sensor probe was brought in contact with the microneedles in the vertical direction at a speed of $0.1 \mathrm{~mm} \mathrm{~s}^{-1}$. The initial distance between the sensor and microneedle tips was set to be $1 \mathrm{~cm}$. Displacement-force measurements were acquired from the point at which the sensor first touched the microneedle tips to the onset of buckling of the microneedles.

Skin penetration efficiency test. Mouse skin after administration of microneedle patch was imaged by digital camera to assess skin penetration efficiency. After administration of microneedle patch for $15 \mathrm{~min}$, mouse ventral skin was stained with trypan blue for $20 \mathrm{~min}$. The mouse was subsequently euthanized, and the skin sample was imaged by digital camera after wiping remaining dye from the skin. In a separate experiment, after $15 \mathrm{~min}$ of administration of microneedle patch, the mouse was euthanized, and its ventral skin was isolated carefully. These excised skins were washed with deionized water and then fixed with $4 \%$ formalin solution for H\&E staining. Histological examination was conducted by an optical microscope under bright-field illumination (Biotek Lionheart FX)

Protein retention test. To access stability of protein bound on the microneedles, the microneedles were coated with BSA-CW800. After washing with PBST and drying, fluorescence images were recorded using LI-COR Odyssey CLx imaging system. Subsequently, microneedles were administered on mouse dorsal skin and left for different periods of time, varying from $15 \mathrm{~s}$ to $60 \mathrm{~min}$. Fluorescence images were recorded again using the same parameters in LI-COR Odyssey CLx imaging system. The fluorescence intensity before and after administration was compared to assess the retained fraction of the protein on the microneedle surface. 
Assessing in vitro biocompatibility of microneedle patch. To evaluate toxicity of microneedles in vitro, HDFs were selected as a model system. Uncoated microneedles and BSA-coated microneedles were incubated in cell culture medium for $1 \mathrm{~h}$ and $16 \mathrm{~h}$, representing short-term and potentially long-term contact with skin tissues. After removing the microneedle patch, HDFs were cultured in the medium for $24 \mathrm{~h}$. Cell viability was quantified using the MTS assay.

Assessing systemic toxicity of microneedle patch. For toxicity assessment in vivo, mice administered with BSA-coated microneedle patch were selected as the treatment group, while mice without administration were used as control group. The representative organs including heart, lung, liver, kidney and spleen in each group were collected and fixed in $4 \%$ neutral buffered formalin for histological analysis, which were subsequently processed by The Musculoskeletal Histology and Morphometry Core at Washington University in St Louis for paraffin embedding, sectioning and H\&E staining. Histological examination was performed with an optical microscope under bright-field illumination (Biotek Lionheart FX).

Bacterial endotoxin level of microneedle patch. To evaluate bacterial endotoxin level on microneedle surface, especially after blocking with BSA, microneedle patches coated with BSA were first incubated with endotoxin-free water overnight. Subsequently, the bacterial endotoxin level in the water was detected and measured by Kinetic Chromogenic Limulus Amebocyte Lysate (LAL) assay (Thermo Scientific, 88282).

Mouse IL-6 ELISA on microneedle and on microtitre plate. Mouse IL-6 DuoSet ELISA kit (R\&D systems, DY406, lot number P195781) was used in the study. Microneedle patches were first placed in a 24-well plate (Corning, 3526) which was clamped on a magnetic plate separator (Luminex Corporation, $\mathrm{CN}-0269$ 01) designed to hold the microplate. Microneedle patches were immersed and incubated with $1 \mathrm{ml}$ of capture antibodies $\left(2 \mu \mathrm{g} \mathrm{ml}^{-1}\right.$ in PBS; R\&D Systems, 840171) overnight at room temperature, followed by washing and blocking with $2 \mathrm{ml}$ reagent diluent ( $1 \times$ PBS containing $3 \%$ BSA, $0.2 \mu \mathrm{m}$ filtered). During incubation and washing steps, microneedle patches were tightly attached to the bottom of well. After three washes with PBST, $1 \mathrm{ml}$ of serial diluted standard samples (R\&D systems, 840173) were added to different wells and microneedle patches were incubated at room temperature for $2 \mathrm{~h}$. Subsequently, patches were washed and incubated with biotinylated detection antibodies (R\&D Systems, 840172, $75 \mathrm{ng} \mathrm{ml}^{-1}$ in reagent diluent) for $2 \mathrm{~h}$, washed again with PBST, and incubated with HRP-labelled streptavidin (R\&D Systems, 893975; 40-fold dilution using reagent diluent) for $20 \mathrm{~min}$. One millilitre of substrate solution (1:1 mixture of colour reagent $\mathrm{A}\left(\mathrm{H}_{2} \mathrm{O}_{2}\right)$ and colour reagent $\mathrm{B}$ (tetramethylbenzidine) (R\&D Systems, DY999)) was added to each well and the reaction was stopped by adding $500 \mu \mathrm{l}$ $\mathrm{H}_{2} \mathrm{SO}_{4}(2 \mathrm{~N})$ (R\&D Systems, DY994) after $20 \mathrm{~min}$. Optical density of each well was determined immediately using a microplate reader set to $450 \mathrm{~nm}$, after removing microneedle patch from each well. IL-6 ELISA on 96-well plate was implemented in the same manner.

Mouse IL-6 FLISA and p-FLISA on microneedle. Mouse IL-6 FLISA was implemented adopting the similar approach as the ELISA described above, except that HRP-labelled streptavidin was replaced with $800 \mathrm{CW}$-labelled streptavidin (LI-COR, 926-32230; $20 \mathrm{ng} \mathrm{ml}^{-1}$ for $20 \mathrm{~min}$ ). The patches were washed three times each with PBST followed by nanopure water. In case of p-FLISA, $1 \mathrm{ml}$ plasmonic fluor-800CW was added subsequently (extinction $\sim 0.5$ ), incubated for $30 \mathrm{~min}$, and the patches were washed 3 times each with reagent diluent followed by PBST. Patches were imaged using a LI-COR CLx fluorescence imager with the following scanning parameters: laser power $\sim \mathrm{L} 2$; resolution $\sim 21 \mu \mathrm{m}$; channel: 800 ; height: $0 \mathrm{~mm}$

Detection of cocaine-specific antibody in an immunized mouse model. Animals and cocaine immunization. Male mice (C57BL/6, Jackson Laboratories 000664), at the age of 5-6 weeks, were purchased from Jackson Laboratories. Mice were housed 4 per cage and allowed to acclimate for $7 \mathrm{~d}$ before the experiment in the housing facility. Eleven mice were randomly divided into two groups, vaccination group and control group. For each mouse in vaccination group, cocaine vaccination solution including $100 \mu \mathrm{g}$ cocaine-BSA conjugate (Fitzgerald Industries, $80-1037$ ) and $2 \mu \mathrm{g}$ LPS (InvivoGen, vac-3pelps) in $100 \mu \mathrm{l}$ saline mixed with $100 \mu \mathrm{l} \%$ Alhydrogel (InvivoGen, vac-alu-250) was freshly prepared before vaccination. Each mouse was first vaccinated subcutaneously with a total of $200 \mu \mathrm{l}$ of cocaine vaccine solution on 4 injection sites and boosted with half the total dosage on 2 sites at day 14, 21 and 28, both on the dorsal side. Two weeks after the last boost, blood from both vaccinated and control group was collected via the submandibular vein and the serum was stored at $-20^{\circ} \mathrm{C}$.

Detection of anti-cocaine antibody in mouse serum. Ninety-six-well ELISA plates (Thermo Scientific, 15041) were coated with $1 \mu \mathrm{g} \mathrm{ml}^{-1}$ BSA or cocaine-BSA conjugate in PBS at $4^{\circ} \mathrm{C}$ overnight and blocked with $300 \mu$ l of Odyssey Blocking Buffer for $1 \mathrm{~h}$. Serum was diluted in PBST at a range of dilution in duplicates and applied on both BSA and cocaine-BSA conjugate coated wells for $1 \mathrm{~h}$ at room temperature. After washing with PBST, plate was incubated with a biotin-labelled donkey anti-mouse antibody (R\&D systems, BAF018; 1:2,000 in 1\% BSA-PBST, $100 \mu \mathrm{l}$ per well) for another $1 \mathrm{~h}$, followed by HRP-labelled streptavidin (R\&D systems, 893975 ; 40 -fold dilution) for $20 \mathrm{~min}$. One-hundred microlitres of substrate solution (1:1 mixture of colour reagent $\mathrm{A}\left(\mathrm{H}_{2} \mathrm{O}_{2}\right)$ and colour reagent $\mathrm{B}$ (tetramethylbenzidine) (R\&D Systems, DY999)) was added to each well and the reaction was stopped with $50 \mu \mathrm{H}_{2} \mathrm{SO}_{4}(2 \mathrm{~N})$ (R\&D Systems, DY994) and absorbance was measured at $450 \mathrm{~nm}$. Within the vaccination group, anti-cocaineBSA titre of each mouse was defined as three mean $+3 \sigma$ of the anti-BSA titre. Mice with higher anti-cocaine-BSA antibody titre than the anti-BSA titre were defined as 'vaccine responders'

In situ sampling and quantification of cocaine-specific antibody in mouse dermal ISF through microneedles. Studying effect of administration time on antibody detection. Microneedle patches were incubated with BSA $\left(1 \mu \mathrm{g} \mathrm{ml}^{-1}\right.$ in PBS) in a 24-well plate at room temperature overnight, followed by washing and blocking with Odyssey Blocking Buffer for $1 \mathrm{~h}$. After three washes with PBST and blow drying with nitrogen gas, microneedle patches were administered on the dorsal skin of the mouse (vaccine responder) under anaesthesia. The period of administration varied from $30 \mathrm{~s}$ to $5 \mathrm{~min}$. Subsequently, patches were washed with PBST and blocked with Odyssey Blocking Buffer for another $30 \mathrm{~min}$, followed by incubation with biotin-labelled donkey anti-mouse antibody (1:2,000 in 1\% BSA-PBST) for $1 \mathrm{~h}$ and $800 \mathrm{CW}$-labelled streptavidin $\left(20 \mathrm{ng} \mathrm{ml}^{-1}\right)$ for $20 \mathrm{~min}$. One millilitre of plasmonic fluor-800CW (extinction $\sim 0.5$ ) was subsequently added, incubated for $30 \mathrm{~min}$, and the patches were washed 3 times each with PBST. Patches were imaged with a LI-COR CLx fluorescence imager with the following scanning parameters: laser power $\sim \mathrm{L} 2$; resolution $\sim 21 \mu \mathrm{m}$; channel: 800 ; height: $0 \mathrm{~mm}$.

Studying the effect of administration location on antibody detection. Eight microneedle patches were incubated with BSA $\left(1 \mu \mathrm{g} \mathrm{ml}^{-1}\right.$ in PBS) in a 24 -well plate at room temperature overnight, followed by washing and blocking with Odyssey Blocking Buffer for $1 \mathrm{~h}$. After three washes with PBST and drying under a stream of nitrogen gas, four microneedle patches were administered on dorsal skin and another four patches were administered on ventral skin of mice for 30 s, simultaneously. Subsequently, patches were washed with PBST and blocked with Odyssey Blocking Buffer for another $30 \mathrm{~min}$, followed by incubation with biotin-labelled donkey anti-mouse antibody (1:2,000 in 1\% BSA-PBST) for $1 \mathrm{~h}$ and $800 \mathrm{CW}$-labelled streptavidin $\left(20 \mathrm{ng} \mathrm{ml}^{-1}\right)$ for $20 \mathrm{~min}$. One millilitre plasmonic fluor-800CW (extinction $~ 0.5$ ) was added subsequently, incubated for $30 \mathrm{~min}$, and the patches were washed 3 times each with PBST. Patches were imaged with a LI-COR CLx fluorescence imager with the following scanning parameters: laser power $\sim \mathrm{L} 2$; resolution $\sim 21 \mu \mathrm{m}$; channel: 800 ; height: $0 \mathrm{~mm}$. The intensity of fluorescence signal represents the amount of anti-BSA antibody binding to the BSA on microneedle surface.

Detection of anti-cocaine antibody through microneedles. Microneedle patches were incubated with BSA or BSA-cocaine conjugate $\left(1 \mu \mathrm{g} \mathrm{ml}^{-1}\right.$ in PBS) in a 24-well plate at room temperature overnight, followed by washing and blocking with Odyssey Blocking Buffer for $1 \mathrm{~h}$. After washing and drying, two microneedle patches coated with BSA and BSA-cocaine conjugate were simultaneously administered on dorsal skin of mouse under anaesthesia for $30 \mathrm{~s}$. Subsequently, microneedle patches were washed and blocked, followed by incubation with anti-mouse antibody for $1 \mathrm{~h}$ and $800 \mathrm{CW}$-labelled streptavidin for $20 \mathrm{~min}$. One millilitre plasmonic fluor-800CW was subsequently added (extinction $\sim 0.5$ ) and incubated for $1 \mathrm{~h}$. Patches were imaged with a LI-COR CLx fluorescence imager using the same scanning parameters as stated above.

\section{Detection and quantification of cytokines in a mouse model of endotoxin} shock. Animals and induction of endotoxin shock. Female mice (BALB/C, Jackson Laboratories, 000651), at the age of 5-6 weeks, were purchased from Jackson Laboratories. Mice were housed 3 per cage and allowed to acclimate for 7 days in the housing facility before the microneedle experiment. To induce endotoxin shock, mice received intraperitoneal injection of LPS (InvivoGen, vac-3pelps; $1 \mathrm{mg} \mathrm{kg}^{-1}$ ), whereas control group mice received intraperitoneal injections of saline solution.

In situ sampling and quantification of mouse IL-6 in dermal ISF through plasmonic fluor-enhanced microneedles. To sample IL-6 in mouse dermal ISF, microneedle patches were pre-functionalized with IL- 6 capture antibody, followed by washing with PBST and blocking with reagent diluent $(1 \times$ PBS containing $3 \%$ BSA, $0.2 \mu \mathrm{m}$ filtered) for $1 \mathrm{~h}$. Microneedle patches were administered on mice ventral skin under anaesthesia for $20 \mathrm{~min}$ to obtain baseline IL-6 measurements. Subsequently, mice were injected with LPS and microneedle patches were administered on mice at 1 , 2.5 and $4 \mathrm{~h}$ after injection in a successive manner. Following $30 \mathrm{~min}$ of blocking with reagent diluent ( $1 \times$ PBS containing $3 \%$ BSA, $0.2 \mu \mathrm{m}$ filtered), microneedle patches were exposed to biotinylated detection antibody, streptavidin-800CW and plasmonic fluor as described above. Fluorescence images of microneedle patches were obtained using a LI-COR CLx fluorescence imager using the same scanning parameters described above. Blood was collected via the submandibular vein immediately after the removal of microneedle patch at last time point ( $4 \mathrm{~h}$ after 
LPS injection) and serum was stored at $-20^{\circ} \mathrm{C}$ for further analysis. IL-6 ELISA was performed as described above in a 96-well microtitre plate to determine the concentration of IL-6 in mouse serum.

To investigate the relationship between serum and ISF at early time points, we used another three groups of mice (randomly divided from 9 mice), administered microneedle patches and collected blood at earlier time points, namely before LPS injection, and 1 and $2.5 \mathrm{~h}$ after LPS injection, respectively. Blood was collected via the submandibular vein right after the removal of microneedle patch at each time point and serum was stored at $-20^{\circ} \mathrm{C}$ for further analysis. Methods and reagents to determine the concentration of IL- 6 in ISF and serum are identical to those mentioned in the previous section. On the basis of the standard curve, concentration of IL- 6 in mouse dermal ISF exhibited a gradual increase from before the LPS injection to $2.5 \mathrm{~h}$ after LPS injection. The IL- 6 concentration in sera also exhibited a similar increase in concentration from before LPS injection to $2.5 \mathrm{~h}$ after LPS injection, in agreement with that observed using the microneedle method (Supplementary Fig. 16).

\section{Specificity of the IL-6 test. It is possible that the fluorescence signals on} microneedles are generated by other cytokines or chemokines whose concentration also increase after LPS injection. To investigate the specificity of the test, we incubated different concentrations of human IL-6, mouse IFN- $\gamma$, mouse TNF, mouse IL-12, mouse CXCL9, mouse CCL19 and mouse CCL20 with mouse IL- 6 capture antibody, followed by mouse IL- 6 detection antibody and HRP or plasmonic fluor (concentration and source of each protein were listed in Supplementary Table 2). For conventional plate-based ELISA, p-FLISA and microneedle-based p-FLISA, assay methods and reagents were identical to those mentioned above.

ELISA (Supplementary Fig. 17a) and p-FLISA (Supplementary Fig. 17b) implemented in microtitre plates indicate that other mouse cytokines or chemokines and IL- 6 from other species do not interfere with the quantification of mouse IL-6. Different concentrations of these interfering proteins exhibited signals similar to those of the blank. In addition, p-FLISA performed on microneedles (Supplementary Fig. 17c) also exhibited weak signals (similar to or lower than the blank) corresponding to interference proteins. Note that the $y$ axis in Supplementary Fig. 17c uses a logarithmic scale.

Detection and quantification of endogenous matricellular protein periostin in periosteum and skin. Animals. Postn ${ }^{K O}$ mice and WT littermate controls on a mixed B6;129 background (Postn ${ }^{\text {tm1Imol }}$, Jackson Laboratories 009067) were a gift from M. F. Rai (Department of Orthopedic Surgery, Washington University in St Louis). Two WT male and two Post ${ }^{K O}$ male mice were used for the plasmonic fluor-enhanced microneedle detection of periostin at the age of 12 weeks.

Histology and immunohistochemistry. The calvaria of WT and Post ${ }^{K O}$ mice were processed by the Musculoskeletal Histology and Morphometry Core at Washington University in St Louis for paraffin embedding, sectioning and H\&E staining. Unstained tissue slides were acquired from the core for periostin immunostaining. In brief, antigen retrieval was performed in sodium citrate buffer $(10 \mathrm{mM}$ sodium citrate, $0.05 \%$ Tween $20, \mathrm{pH} 6.0$ ) at $50-55^{\circ} \mathrm{C}$ overnight. The tissues were then permeabilized in $0.2 \%$ Triton X-100 buffer (Sigma Aldrich 9002-93-1) for $10 \mathrm{~min}$, blocked in 2.5\% normal horse serum (IMMPRESS HRP Anti-Rabbit IgG kit, Vector Laboratories, MP-7401) for $1 \mathrm{~h}$ at room temperature, and incubated with anti-periostin antibody (Sigma Aldrich, HPA012306) with a dilution rate of 1:200 at $4{ }^{\circ} \mathrm{C}$ overnight. The endogenous peroxidase was quenched in $0.3 \% \mathrm{H}_{2} \mathrm{O}_{2}$ for $30 \mathrm{~min}$ and the sections were incubated with biotinylated secondary antibody (IMMPRESS HRP Anti-Rabbit IgG kit, Vector Laboratories, MP-7401-15) for $30 \mathrm{~min}$ at room temperature and the stain was developed by incubating with metal enhanced DAB substrate (IMMPACT DAB kit, Vector Laboratories, SK-4105). Nuclei were counterstained with haematoxylin (Ricca chemical 3536-16). All washes between steps were performed in either double-distilled water or TNT buffer (0.1 M Tris-HCl, 0.15 M NaCl, $0.05 \%$ Tween)

In situ sampling and quantification of periostin in mouse periosteum and skin through plasmonic fluor-enhanced microneedle. Mouse periostin Duoset ELISA kit (R\&D Systems, DY2955, lot number P217047) was used. To sample periostin in mouse periosteum and dermal ISF, microneedle patches were pre-functionalized with periostin capture antibody (R\&D Systems, 842318) in a 24-well plate clamped on a magnetic plate separator, followed by washing with PBST and blocking with reagent diluent ( $1 \times$ PBS containing $3 \%$ BSA, $0.2 \mu \mathrm{m}$ filtered). Skin above periosteum the layer was carefully incised using scissors. A microneedle patch was administered on periosteum and another patch was administered on dorsal skin for $10 \mathrm{~min}$, under anaesthesia. This was followed by another $30 \mathrm{~min}$ of blocking with reagent diluent ( $1 \times$ PBS containing $3 \% \mathrm{BSA}$, $0.2 \mu \mathrm{m}$ filtered), microneedle patches were exposed to biotinylated detection antibody (R\&D Systems, 842319), streptavidin-800CW and plasmonic fluor. Fluorescence maps of microneedle patches were imaged using a LI-COR CLx fluorescence imager using the same scanning parameters as described above. Blood was collected by cardiac puncture and serum was stored at $-20^{\circ} \mathrm{C}$ for further analysis. Periostin ELISA for mouse serum were implemented in similar approach on 96 -well microtitre plate.
Statistics. To analyse the statistical difference between two groups, unpaired one-tailed $t$-test with Welch's correction was used. To analyse the statistical difference between each data point in two groups, two-way ANOVA with Sidak's multiple-comparison test was used. For analysing the statistical difference between two or more groups one-way ANOVA with Tukey's multiple-comparison test was used. Statistical significance of the data was calculated at 95\% $(P<0.05)$ confidence intervals. All values are expressed as mean \pm s.d. GraphPad Prism 8 was used for all statistical analysis. We used four-parameter logistic or polynomial fit to calculate the LOD in the standard curves of bioassays. The LOD is defined as the analyte concentration corresponding to the mean fluorescence intensity of blank plus $3 \sigma$. Origin 2016 was used to calculate the LOD.

Reporting Summary. Further information on research design is available in the Nature Research Reporting Summary linked to this article.

\section{Data availability}

The main data supporting the results in this study are available within the paper and its Supplementary Information. The raw data are available from figshare at https://doi.org/10.6084/m9.figshare.13331366.

Received: 4 May 2020; Accepted: 4 December 2020; Published online: 22 January 2021

\section{References}

1. Kool, J. et al. Suction blister fluid as potential body fluid for biomarker proteins. Proteomics 7, 3638-3650 (2007).

2. Müller, A. C. et al. A comparative proteomic study of human skin suction blister fluid from healthy individuals using immunodepletion and iTRAQ labeling. J. Proteome Res. 11, 3715-3727 (2012).

3. Tran, B. Q. et al. Proteomic characterization of dermal interstitial fluid extracted using a novel microneedle-assisted technique. J. Proteome Res. 17, 479-485 (2018)

4. Heikenfeld, J. et al. Accessing analytes in biofluids for peripheral biochemical monitoring. Nat. Biotechnol. 37, 407-419 (2019).

5. He, R. et al. A hydrogel microneedle patch for point-of-care testing based on skin interstitial fluid. Adv. Healthc. Mater. 9, 1901201 (2020).

6. Wang, Z. et al. Transdermal colorimetric patch for hyperglycemia sensing in diabetic mice. Biomaterials 237, 119782 (2020).

7. Gromov, P. et al. Tumor interstitial fluid-a treasure trove of cancer biomarkers. Biochim. Biophys. Acta 1834, 2259-2270 (2013).

8. Yang, B., Fang, X. \& Kong, J. In situ sampling and monitoring cell-free DNA of the Epstein-Barr virus from dermal interstitial fluid using wearable microneedle patches. ACS Appl. Mater. Interfaces 11, 38448-38458 (2019).

9. Al Sulaiman, D. et al. Hydrogel-coated microneedle arrays for minimally invasive sampling and sensing of specific circulating nucleic acids from skin interstitial fluid. ACS Nano 13, 9620-9628 (2019).

10. McHugh, K. J. et al. Biocompatible near-infrared quantum dots delivered to the skin by microneedle patches record vaccination. Sci. Transl. Med. 11, eaay7162 (2019).

11. Chang, $H$. et al. A swellable microneedle patch to rapidly extract skin interstitial fluid for timely metabolic analysis. Adv. Mater. 29, 1702243 (2017)

12. Kiistala, U. Suction blister device for separation of viable epidermis from dermis. J. Invest Dermatol. 50, 129-137 (1968).

13. Krogstad, A., Jansson, P. A., Gisslen, P. \& Lönnroth, P. Microdialysis methodology for the measurement of dermal interstitial fluid in humans. Br. J. Dermatol. 134, 1005-1012 (1996).

14. Bodenlenz, M. et al. Open flow microperfusion as a dermal pharmacokinetic approach to evaluate topical bioequivalence. Clin. Pharmacokinet. 56, 91-98 (2017).

15. Samant, P. P. \& Prausnitz, M. R. Mechanisms of sampling interstitial fluid from skin using a microneedle patch. Proc. Natl Acad. Sci. USA 115, 4583-4588 (2018)

16. Taylor, R. M., Miller, P. R., Ebrahimi, P., Polsky, R. \& Baca, J. T. Minimally-invasive, microneedle-array extraction of interstitial fluid for comprehensive biomedical applications: transcriptomics, proteomics, metabolomics, exosome research, and biomarker identification. Lab. Anim. 52, 526-530 (2018).

17. Muller, D. A., Corrie, S. R., Coffey, J., Young, P. R. \& Kendall, M. A. Surface modified microprojection arrays for the selective extraction of the dengue virus NS1 protein as a marker for disease. Anal. Chem. 84, 3262-3268 (2012).

18. Coffey, J. W., Meliga, S. C., Corrie, S. R. \& Kendall, M. A. Dynamic application of microprojection arrays to skin induces circulating protein extravasation for enhanced biomarker capture and detection. Biomaterials $\mathbf{8 4}$, 130-143 (2016).

19. Nedrebø, T., Reed, R. K., Jonsson, R., Berg, A. \& Wiig, H. Differential cytokine response in interstitial fluid in skin and serum during experimental inflammation in rats. J. Physiol. 556, 193-202 (2004). 
20. Zhang, X., Chen, G., Bian, F., Cai, L. \& Zhao, Y. Encoded microneedle arrays for detection of skin interstitial fluid biomarkers. Adv. Mater. 31, 1902825 (2019).

21. Coffey, J. W., Corrie, S. R. \& Kendall, M. A. Rapid and selective sampling of IgG from skin in less than 1 min using a high surface area wearable immunoassay patch. Biomaterials 170, 49-57 (2018).

22. Luan, J. et al. Ultrabright fluorescent nanoscale labels for the femtomolar detection of analytes with standard bioassays. Nat. Biomed. Eng. 4, 518-530 (2020).

23. Dulkeith, E. et al. Gold nanoparticles quench fluorescence by phase induced radiative rate suppression. Nano Lett. 5, 585-589 (2005).

24. Davis, S. P., Landis, B. J., Adams, Z. H., Allen, M. G. \& Prausnitz, M. R. Insertion of microneedles into skin: measurement and prediction of insertion force and needle fracture force. J. Biomech. 37, 1155-1163 (2004).

25. Kampman, K. M. The treatment of cocaine use disorder. Sci. Adv. 5, eaax1532 (2019).

26. Shorter, D. \& Kosten, T. R. Novel pharmacotherapeutic treatments for cocaine addiction. BMC Med. 9, 119 (2011).

27. Kinsey, B. M., Kosten, T. R. \& Orson, F. M. Anti-cocaine vaccine development. Expert Rev. Vaccines 9, 1109-1114 (2010).

28. Martell, B. et al. Cocaine vaccine for the treatment of cocaine dependence: a randomized double-blind placebo-controlled efficacy trial. Arch. Gen. Psych. 66, 1116-1123 (2009).

29. Scott, E. A., Karabin, N. B. \& Augsornworawat, P. Overcoming immune dysregulation with immunoengineered nanobiomaterials. Annu. Rev. Biomed. Eng. 19, 57-84 (2017).

30. Taylor, J., Laudenbach, M., Tucker, A., Jenkins, M. \& Pravetoni, M. Hapten-specific naive B cells are biomarkers of vaccine efficacy against drugs of abuse. J. Immunol. Methods 405, 74-86 (2014).

31. Monteiro-Riviere, N. A., Bristol, D. G., Manning, T. O., Rogers, R. A. \& Riviere, J. E. Interspecies and interregional analysis of the comparative histologic thickness and laser Doppler blood flow measurements at five cutaneous sites in nine species. J. Invest. Dermatol. 95, 582-6 (1990).

32. Davidson, A., Al-Qallaf, B. \& Das, D. B. Transdermal drug delivery by coated microneedles: geometry effects on effective skin thickness and drug permeability. Chem. Eng. Res. Des. 86, 1196-1206 (2008).

33. Copeland, S., Warren, H. S., Lowry, S. F., Calvano, S. E. \& Remick, D. Acute inflammatory response to endotoxin in mice and humans. Clin. Diagn. Lab. Immunol. 12, 60-67 (2005).

34. Hong, Y.-H., Chao, W.-W., Chen, M.-L. \& Lin, B.-F. Ethyl acetate extracts of alfalfa (Medicago sativa L.) sprouts inhibit lipopolysaccharide-induced inflammation in vitro and in vivo. J. Biomed. Sci. 16, 64 (2009).

35. Colnot, C. Skeletal cell fate decisions within periosteum and bone marrow during bone regeneration. J. Bone Miner. Res. 24, 274-282 (2009).

36. Dwek, J. R. The periosteum: what is it, where is it, and what mimics it in its absence? Skelet. Radiol. 39, 319-323 (2010).

37. Sakai, D. et al. Remodeling of actin cytoskeleton in mouse periosteal cells under mechanical loading induces periosteal cell proliferation during bone formation. PLoS ONE 6, e24847 (2011)

38. Moore, S. R., Milz, S. \& Knothe Tate, M. L. Periosteal thickness and cellularity in mid-diaphyseal cross-sections from human femora and tibiae of aged donors. J. Anat. 224, 142-149 (2014).

39. Merle, B. \& Garnero, P. The multiple facets of periostin in bone metabolism Osteoporos. Int. 23, 1199-1212 (2012).

40. Kyutoku, M. et al. Role of periostin in cancer progression and metastasis: inhibition of breast cancer progression and metastasis by anti-periostin antibody in a murine model. Int. J. Mol. Med. 28, 181-186 (2011).

41. Yan, J. et al. Circulating periostin levels increase in association with bone density loss and healing progression during the early phase of hip fracture in Chinese older women. Osteoporos. Int. 28, 2335-2341 (2017).
42. Bonnet, N., Garnero, P. \& Ferrari, S. Special Issue on bone disease mechanisms: periostin action in bone. Mol. Cell. Endocrinol. 432, 75-82 (2015).

43. Cai, Y. et al. Magnet patterned superparamagnetic $\mathrm{Fe}_{3} \mathrm{O}_{4} / \mathrm{Au}$ core-shell nanoplasmonic sensing array for label-free high throughput cytokine immunoassay. Adv. Healthc. Mater. 8, 1801478 (2019).

44. $\mathrm{Hu}, \mathrm{M}$. et al. Gold nanostructures: engineering their plasmonic properties for biomedical applications. Chem. Soc. Rev. 35, 1084-1094 (2006)

45. Lee, K.-S. \& El-Sayed, M. A. Dependence of the enhanced optical scattering efficiency relative to that of absorption for gold metal nanorods on aspect ratio, size, end-cap shape, and medium refractive index. J. Phys. Chem. B 109, 20331-20338 (2005)

46. Gole, A. \& Murphy, C. J. Azide-derivatized gold nanorods: functional materials for 'click' chemistry. Langmuir 24, 266-272 (2008).

47. Tebbe, M., Kuttner, C., Männel, M., Fery, A. \& Chanana, M. Colloidally stable and surfactant-free protein-coated gold nanorods in biological media. ACS Appl. Mater. Interfaces 7, 5984-5991 (2015).

\section{Acknowledgements}

We acknowledge support from the National Science Foundation (CBET-1900277) and the National Institutes of Health (R01DE027098, R56DE027924, R01CA141521, R21DA036663, R21CA236652). We thank the Nano Research Facility and Institute of Materials Science and Engineering at Washington University for providing access to electron microscopy facilities, N. Huebsch for providing access to the fluorescence microscope, K. Magee for the help with mouse experiments, Y. Diao for help with digital photographs and $\mathrm{M}$. Shen for inspiring discussions.

\section{Author contributions}

S.S., Z.W. and J.L. conceived the project. S.S., J.S.R., E.L.S., Z.W. and J.L. designed the experiments. Z.W., J.L. and M.Y. fabricated the microneedle patches. Z.W., A.S., L.L., X.Z., J.S.R. and E.L.S. performed the animal experiments. Z.W., J.L., A.S., L.L., Q.Z. and J.J.M. performed the bioassays. J.L. and Q.J. synthesized the plasmonic fluors. P.G. synthesized the magnetic nanoparticles. R.G. performed TEM imaging. P.R. and Y.W. performed SEM imaging. S.C. collected fluorescence images of the microneedles. S.S. J.S.R., E.L.S., J.J.M., Z.W. and J.L. wrote the paper. All authors reviewed and commented on the manuscript.

\section{Competing interests}

The authors declare the following competing financial interests: J.L., J.J.M. and S.S. are inventors on a provisional patent related to plasmonic fluor technology and the technology has been licensed by the Office of Technology Management at Washington University in St Louis to Auragent Bioscience LLC, which is developing plasmonic fluor products. J.L., J.J.M. and S.S. are co-founders and shareholders of Auragent Bioscience LLC. These potential conflicts of interest have been disclosed and are being managed by Washington University in St Louis.

\section{Additional information}

Supplementary information is available for this paper at https://doi.org/10.1038/ s41551-020-00672-y.

Correspondence and requests for materials should be addressed to E.L.S., J.S.R. or S.S. Peer review information Peer reviewer reports are available.

Reprints and permissions information is available at www.nature.com/reprints.

Publisher's note Springer Nature remains neutral with regard to jurisdictional claims in published maps and institutional affiliations.

(c) The Author(s), under exclusive licence to Springer Nature Limited 2021 


\section{natureresearch}

Corresponding author(s): Srikanth Singamaneni, Erica Scheller, Jai Rudra Last updated by author(s): Dec 1, 2020

\section{Reporting Summary}

Nature Research wishes to improve the reproducibility of the work that we publish. This form provides structure for consistency and transparency in reporting. For further information on Nature Research policies, see Authors \& Referees and the Editorial Policy Checklist.

\section{Statistics}

For all statistical analyses, confirm that the following items are present in the figure legend, table legend, main text, or Methods section.

n/a Confirmed

$\bigotimes$ The exact sample size $(n)$ for each experimental group/condition, given as a discrete number and unit of measurement

$\bigotimes$ A statement on whether measurements were taken from distinct samples or whether the same sample was measured repeatedly

The statistical test(s) used AND whether they are one- or two-sided

Only common tests should be described solely by name; describe more complex techniques in the Methods section.

Х $\square$ A description of all covariates tested

\ $\square$ A description of any assumptions or corrections, such as tests of normality and adjustment for multiple comparisons

A full description of the statistical parameters including central tendency (e.g. means) or other basic estimates (e.g. regression coefficient)

$\triangle$ AND variation (e.g. standard deviation) or associated estimates of uncertainty (e.g. confidence intervals)

For null hypothesis testing, the test statistic (e.g. $F, t, r$ ) with confidence intervals, effect sizes, degrees of freedom and $P$ value noted

Give $P$ values as exact values whenever suitable.

Х $\square$ For Bayesian analysis, information on the choice of priors and Markov chain Monte Carlo settings

Х $\square$ For hierarchical and complex designs, identification of the appropriate level for tests and full reporting of outcomes

Х $\square$ Estimates of effect sizes (e.g. Cohen's $d$, Pearson's $r$ ), indicating how they were calculated

Our web collection on statistics for biologists contains articles on many of the points above.

\section{Software and code}

\section{Policy information about availability of computer code}

Data collection Licor Image Studio ver 5.2, UV Probe 2.43, Biotek Lionheart FX software, Bruker D8-Advance X-ray powder diffractometer, Olympus FV1000 confocal-microscope software

Data analysis Graphpad Prism 8, Origin 2016, Image J 1.6.0_24, Licor Image Studio Lite, FV10-ASW 3.0 Viewer

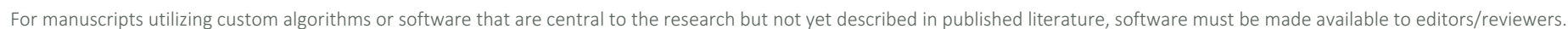
We strongly encourage code deposition in a community repository (e.g. GitHub). See the Nature Research guidelines for submitting code \& software for further information.

\section{Data}

Policy information about availability of data

All manuscripts must include a data availability statement. This statement should provide the following information, where applicable:

- Accession codes, unique identifiers, or web links for publicly available datasets

- A list of figures that have associated raw data

- A description of any restrictions on data availability

The main data supporting the results in this study are available within the paper and its Supplementary Information. The raw data are available from figshare with the identifier https://doi.org/10.6084/m9.figshare.13331366. 
Please select the one below that is the best fit for your research. If you are not sure, read the appropriate sections before making your selection. \Life sciences Behavioural \& social sciences Ecological, evolutionary \& environmental sciences

For a reference copy of the document with all sections, see nature.com/documents/nr-reporting-summary-flat.pdf

\section{Life sciences study design}

All studies must disclose on these points even when the disclosure is negative.

Sample size No statistical method was used to determine sample sizes.

Data exclusions No data were excluded from the analyses.

Replication We have replicated most of the experiments at least twice to support all of the claims made in this manuscript.

Randomization The mice used in each model were chosen randomly.

Blinding The investigator was not blinded to group allocation during the experiments.

\section{Reporting for specific materials, systems and methods}

We require information from authors about some types of materials, experimental systems and methods used in many studies. Here, indicate whether each material, system or method listed is relevant to your study. If you are not sure if a list item applies to your research, read the appropriate section before selecting a response.

Materials \& experimental systems

$\mathrm{n} / \mathrm{a}$ Involved in the study

Methods

$\square$ Antibodies

$\square$ Eukaryotic cell lines

$\mathrm{n} / \mathrm{a}$ Involved in the study

Х Palaeontology

Х ChIP-seq

X $\square$ Flow cytometry

$\square$ Animals and other organisms

Х $\square$ MRI-based neuroimaging

\ $\square$ Human research participants

\ $\square$ Clinical data

\section{Antibodies}

Antibodies used

Mouse IL-6 ELISA kit (R\&D, catalog number DY 406, lot number P195781, including capture antibody (part number 840171), detection antibody (part number 840172). Donkey anti-mouse IgG biotinylated antibody (R\&D, catalog number BAF018). Antiperiostin antibody (Sigma Aldrich HPA012306). ImmPRESS ${ }^{\circledR}$ HRP Horse Anti-Rabbit IgG Polymer Detection Kit (Vector Laboratory, Catalog number MP-7401-15). Mouse periostin ELISA kit (R\&D catalog number DY2955, lot number P217047, including capture antibody (part number 842318), detection antibody (part number 842319)).

Validation

All antibodies used in the study had been tested for proper performance and function under established quality-control testing criteria by the vendors.

\section{Eukaryotic cell lines}

Policy information about cell lines

Cell line source(s)

Human dermal fibroblasts were purchased from ATCC.

Authentication

Human dermal fibroblasts were authenticated using STR analysis provided by ATCC.

Mycoplasma contamination

The cell line tested negative for mycoplasma contamination, according to ATCC.

Commonly misidentified lines

(See ICLAC register)

No commonly misidentified cell lines were used. 
Policy information about studies involving animals; ARRIVE guidelines recommended for reporting animal research

Laboratory animals

Wild animals

Field-collected samples

Ethics oversight
Male mice (C57BL/6), aged 5-6 weeks. Female mice (BALB/C), aged 5-6 weeks. Male mice (B6;129-Postntm1Jmol/J), aged 12 weeks.

The study did not involve wild animals.

The study did not involve samples collected from the field.

IACUC from Washington University in St. Louis.

Note that full information on the approval of the study protocol must also be provided in the manuscript. 